\title{
A dynamic hybrid local/nonlocal continuum model for wave propagation
}

\author{
Fei Han ${ }^{1}$ - Shankun Liu ${ }^{1}$ - Gilles Lubineau ${ }^{2}$
}

Received: 28 November 2018 / Accepted: 14 October 2020

(c) Springer-Verlag GmbH Germany, part of Springer Nature 2020

\begin{abstract}
In this work, we develop a dynamic hybrid local/nonlocal continuum model to study wave propagations in a linear elastic solid. The developed hybrid model couples, in the dynamic regime, a classical continuum mechanics model with a bond-based peridynamic model using the Morphing coupling method that introduced in a previous study (Lubineau et al., J Mech Phys Solids 60(6):1088-1102, 2012). The classical continuum mechanical model is known as a local continuum model, while the peridynamic model is known as a nonlocal continuum model. This dynamic hybrid model aims to introduce the nonlocal model into the key structural domain, in which the dispersions or crack nucleations may occur due to flaws, while applying the local model to the rest of the structural domain. Both the local and nonlocal continuum domains are overlapped in the coupled subdomain. We study the speeds and angular frequencies of the plane waves, with small and large wavenumbers obtained by the hybrid model and compare them to purely local and purely nonlocal solutions. The error of the hybrid model is discussed by analyzing the ghost forces, and the work done by the ghost forces is considered equivalent to the energy of spurious reflections. One- and two-dimensional numerical examples illustrate the validity and accuracy of the proposed approach. We show that this dynamic hybrid local/nonlocal continuum model can be successfully applied to simulate wave propagations and crack nucleations induced by waves.
\end{abstract}

Keywords Peridynamics · Continuum mechanics · Morphing coupling method $\cdot$ Hybrid model $\cdot$ Wave dispersion $\cdot$ Fracture

\section{Introduction}

Peridynamics is a recently developed nonlocal theory in solid mechanics [1]. The peridynamic model redefines mechanical problems by replacing the partial differential equations with integral equations, which can naturally handle discontinuous solutions generated by spatial discontinuities. In doing so, the peridynamic model is an interesting tool for studying cracks and how they propagate. In a peridynamic model, it is assumed that the equilibrium of a material point is attained by an integral of internal forces exerted by surrounding points

Gilles Lubineau

gilles.lubineau@kaust.edu.sa

1 State Key Laboratory of Structural Analysis for Industrial Equipment, Department of Engineering Mechanics, International Research Center for Computational Mechanics, Dalian University of Technology, Dalian 116023, People's Republic of China

2 Physical Science and Engineering Division, COHMAS Laboratory, King Abdullah University of Science and Technology (KAUST), Thuwal 23955-6900, Saudi Arabia across a finite distance [2]. An internal force is defined over the connection vector, called the "bond", between pairs of points. A bond is often set to break irreversibly when it is stretched beyond its critical length [3]. As a result, cracks are represented by domains that are crossed by broken bonds only. This definition of material flaws via broken bonds permits crack initiation and evolution simultaneously at multiple sites and along spontaneous paths, with a simple bond-breaking law rather than a traditional crack-growth law. Thus, peridynamics is promising for the simulation of micro-crack nucleation to macro-crack propagation [4-6], particularly for the concurrence of multiple cracks [3]. This theory's potential has attracted considerable attention in the last decade [7].

However, applying the integral equations at each point makes peridynamics significantly more computationally expensive than classical continuum mechanics. Additionally, the peridynamic method requires boundary conditions to be applied over a thick layer and is not compatible with classical traction-like boundary conditions, which are more standard in routine engineering. Consequently, a reasonable strategy 
is to reserve the peridynamics for severely degraded parts of a structure in which dominant mechanisms strongly affect the solution, such as damage or fracture mechanisms. In contrast, local continuum mechanics is used for the rest of a structure to satisfy boundary conditions and preserve computational efficiency. The challenge then lies in how to efficiently couple the peridynamic model with the classical continuum mechanics model in a variety of situations (quasi-static, dynamics, linear, or non-linear material constitutive responses, etc.).

In recent years, diverse coupling schemes have been proposed to couple the peridynamics with classical continuum mechanics. A recent paper [8] reviewed these local-to-nonlocal coupling methods, such as the discretebased coupling approaches [9-11], the variable-horizon approaches [12], the optimization-based approaches [13], and the continuum-based coupling approaches [14-16]. The continuum-based coupling schemes can be further subdivided into the force-based coupling method [16], the Arlequin coupling method [14], and the Morphing coupling method [15]. The force-based coupling method derives a coupled force equilibrium equation, which blends the peridynamic and classical continuum models into a transition region by using a weighting function. Besides, the Arlequin coupling method belongs to the energy-based coupling strategy in which the coupling is performed at the energy level by using a partition-of-unity concept. By comparison, the Morphing coupling method constructs a single unified balance between constitutive parameters of the peridynamic model and the classical continuum model, using the equivalent energy density of both models. This constitutive constraint can automatically correct the local stiffness tensor to accommodate the coupling function, to guarantee that both coupled models have the uniform effective stiffness that is key to successful coupling. Additionally, this simple balance constraint has no additional parameters like Lagrange multiplies in the Arlequin method [14], which can easily accommodate more complex elasticity such as anisotropy. With the help of this single constraint, the coupling artifacts are analytically studied through estimating the ghost forces in both [15] and this paper, which helps to understand and control the coupling errors. Note that the Morphing coupling method leads to an overlapping domain that has a minimum of two horizons in the span of the domain. Moreover, the assumption of a homogeneous deformation over the neighborhood of the peridynamic point in the overlapping domain is needed for the current Morphing technique, which may face a challenge for the composite and heterogeneous materials.

The Morphing coupling method presents a promising approach because it is simple and robust [15,17-21]. A new way to apply the peridynamics as a regularization tool to the classical continuum damage mechanics through the Morphing techniquehas has been recently developed, which produces a hybrid local/nonlocal continuum model for quasi- static damage and fracture behaviors [20]. However, the proposed hybrid models are only used for static or quasistatic problems. A hybrid local/nonlocal continuum model for a dynamic problem still requires the extension of the Morphing coupling method to this case.

This paper aims to develop a hybrid local/nonlocal continuum equation of motion based on the Morphing coupling method. Using the dynamic hybrid local/nonlocal model, we analyze the relations between the speed and angular frequency of waves. Then, we apply this hybrid equation of motion to simulate the wave propagation in a linear elastic solid so we can focus on well-known artifacts in dynamic problems, such as the false wave produced by the coupling scheme. The dispersion of high-frequency waves (i.e., a wave with large wavenumbers) occurs in the nonlocal peridynamic model, but the dispersion does not occur in the local classical continuum model. Consequently, the propagation of high-frequency waves simulated by the hybrid local/nonlocal models presents another interesting point. Furthermore, using the advantages of peridynamics in simulating crack nucleation and propagation, we also apply the hybrid model to simulate local failure caused by shock loads.

The remainder of this paper is organized as follows: Sect. 2 states the key formulation of the bond-based peridynamics, and Sect. 3 derives the hybrid local/nonlocal equations of motion through the Morphing coupling method. We estimate the speeds of plane harmonic waves and analyze the ghost force and its work in Sect. 4. We study and describe one-dimensional (1D) and two-dimensional (2D) examples in Sect. 5. Concluding remarks are provided in Sect. 6.

\section{Bond-based peridynamic model}

In this section, we will briefly introduce the bond-based peridynamic model, which has been widely described in the literature $[1,2,22,23]$. We consider a complete domain $\Omega$. The bond-based peridynamic equation of motion, which uses a pairwise force function $\boldsymbol{f}$ to describe the interaction between material points, is written as follows:

$$
\rho(\boldsymbol{x}) \ddot{\boldsymbol{u}}(\boldsymbol{x}, t)=\int_{\mathcal{H}_{\delta}(\boldsymbol{x})} \boldsymbol{f}\left(\boldsymbol{x}^{\prime}, \boldsymbol{x}, t\right) d V_{\boldsymbol{x}^{\prime}}+\boldsymbol{b}(\boldsymbol{x}, t) \quad \forall \boldsymbol{x} \in \Omega,
$$

where $t$ denotes the time, $\boldsymbol{b}$ is a prescribed body force density, $\boldsymbol{u}$ is displacement, $\rho$ is the material density, and $\mathcal{H}_{\delta}(\boldsymbol{x})$ is a neighborhood of the point $\boldsymbol{x}$ defined as

$\mathcal{H}_{\delta}(\boldsymbol{x}):=\left\{\boldsymbol{x}^{\prime} \in \mathbb{R}^{d}, d=1,2\right.$, or $\left.3:\left|\boldsymbol{x}^{\prime}-\boldsymbol{x}\right| \leq \delta\right\}$ 


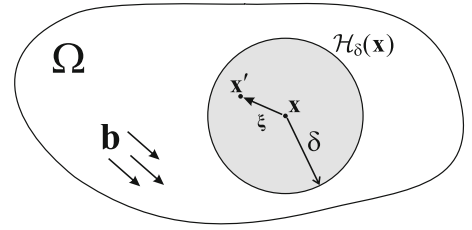

(a)

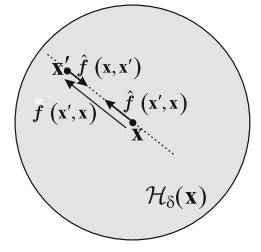

(b)
Fig. 1 a Continuum body $\Omega$ and neighborhood of $\boldsymbol{x}, \mathcal{H}_{\delta}(\boldsymbol{x})$. b Decomposition of the pairwise force function, $\mathbf{f}$, into partial interactions in the spacial dimension

which denotes the action scope of $\boldsymbol{x}$, and $\delta$ is referred to as the peridynamic horizon [1] (see Fig. 1). An interaction between $\boldsymbol{x}$ and another point that is outside of $\mathcal{H}_{\delta}(\boldsymbol{x})$ is truncated.

The pairwise force function is defined as (see [2])

$\boldsymbol{f}\left(\boldsymbol{x}^{\prime}, \boldsymbol{x}, t\right)=\hat{\boldsymbol{f}}\left(\boldsymbol{x}^{\prime}, \boldsymbol{x}, t\right)-\hat{\boldsymbol{f}}\left(\boldsymbol{x}, \boldsymbol{x}^{\prime}, t\right)$,

where $\hat{\boldsymbol{f}}\left(\boldsymbol{x}^{\prime}, \boldsymbol{x}, t\right)$ is the partial interaction due to the action of the point $\boldsymbol{x}^{\prime}$ on the point $\boldsymbol{x}$ at time $t$, and $\hat{\boldsymbol{f}}\left(\boldsymbol{x}, \boldsymbol{x}^{\prime}, t\right)$ is the partial interaction due to the action of the point $x$ on the point $\boldsymbol{x}^{\prime}$ at time $t$. A possible constitutive model for linear elasticity and small deformations is (see [1,2])

$\hat{\boldsymbol{f}}\left(\boldsymbol{x}^{\prime}, \boldsymbol{x}, t\right)=\frac{1}{2} \boldsymbol{C}(\boldsymbol{x}, \boldsymbol{\xi})\left\{\boldsymbol{u}\left(\boldsymbol{x}^{\prime}, t\right)-\boldsymbol{u}(\boldsymbol{x}, t)\right\}$,

where $\boldsymbol{u}$ is displacement, $\boldsymbol{\xi}=\boldsymbol{x}^{\prime}-\boldsymbol{x}$ is the relative position vector in the reference configuration, and $\boldsymbol{C}(\boldsymbol{x}, \boldsymbol{\xi})$ is the second-order micromodulus tensor defined below [1]:

$\boldsymbol{C}(\boldsymbol{x}, \boldsymbol{\xi})=c(\boldsymbol{x}, \boldsymbol{\xi}) \boldsymbol{\xi} \otimes \boldsymbol{\xi}$

where $c(\boldsymbol{x}, \boldsymbol{\xi})$ is the coefficient function.

\section{Dynamic hybrid local/nonlocal continuum model}

In this section, we develop a dynamic hybrid local/nonlocal continuum model using the Morphing coupling method, which has been employed to couple the peridynamic model with the classical continuum mechanics model for static or quasi-static problems $[15,17,19]$.

We divide this domain $\Omega$ into three subdomains: $\Omega_{1}, \Omega_{2}$ and $\Omega_{m}$ (see Fig. 2), i.e., $\Omega=\Omega_{1} \cup \Omega_{2} \cup \Omega_{m}, \Omega_{1} \cap \Omega_{2}=\emptyset$, $\Omega_{1} \cap \Omega_{m}=\emptyset$ and $\Omega_{2} \cap \Omega_{m}=\emptyset$. Here, $\Omega_{2}$ is totally embedded within $\Omega_{m}$, and $\Omega_{m}$ is totally embedded within $\Omega_{1}$, such that $\partial \Omega_{2} \cap \partial \Omega_{1}=\emptyset$ and $\partial \Omega \subset \partial \Omega_{1}$. Therefore, $\Omega_{m}$ becomes a transition domain between $\Omega_{1}$ and $\Omega_{2}$.

Rather than using a peridynamic model in the whole $\Omega$, we proposed a Morphing coupling method through which the peridynamic model is employed only where needed. Here, it

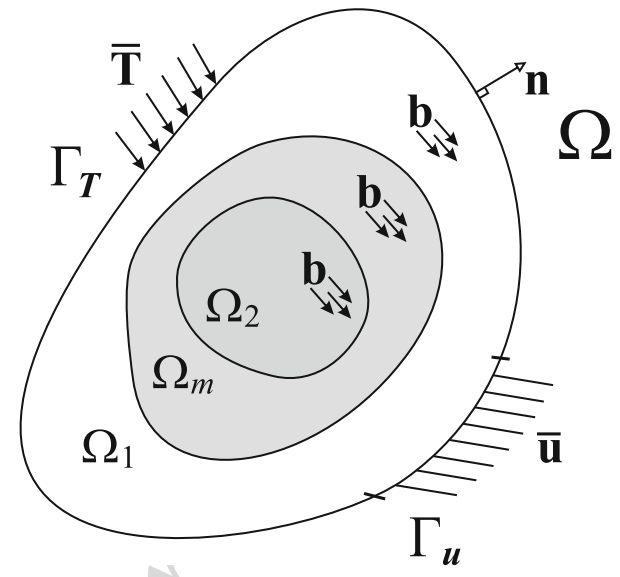

Fig. 2 The whole domain $\Omega$ consists of $\Omega_{1}, \Omega_{2}$ and $\Omega_{m}$

is the subdomain $\Omega_{2}$ in which nonlocal effects, such as wave dispersions or crack nucleations and propagations, need to be simulated. The classical continuum model is prescribed in the subdomain $\Omega_{1}$, where the deformation is considered to be smooth. The morphing between these two models, i.e., the hybrid local/nonlocal model, is constructed in the transition domain $\Omega_{m}$. The displacement, $\overline{\boldsymbol{u}}$, is imposed on the part $\Gamma_{\boldsymbol{u}}$ of $\partial \Omega$, and the traction $\bar{T}$ is imposed on the complementary part $\Gamma_{\boldsymbol{T}}$ of $\partial \Omega . \boldsymbol{n}$ is an outward unit normal. Also, the whole domain $\Omega$ is subjected to body forces denoted by $\boldsymbol{b}$.

The Morphing method begins with a unified system of governing equations in $\Omega$, which builds a hybrid local/nonlocal continuum model as follows. The Morphing technique then leads to a simple evolution of material properties for characterizing each model.

Kinematic admissibility and compatibility

$$
\begin{aligned}
& \boldsymbol{\varepsilon}(\boldsymbol{x}, t)=\frac{1}{2}\left(\nabla \boldsymbol{u}(\boldsymbol{x}, t)+(\nabla \boldsymbol{u})^{\mathrm{T}}(\boldsymbol{x}, t)\right) \quad \forall \boldsymbol{x} \in \Omega \backslash \Omega_{2}, \\
& \boldsymbol{\eta}\left(\boldsymbol{x}^{\prime}, \boldsymbol{x}, t\right)=\boldsymbol{u}\left(\boldsymbol{x}^{\prime}, t\right)-\boldsymbol{u}(\boldsymbol{x}, t) \quad \forall \boldsymbol{x}, \boldsymbol{x}^{\prime} \in \Omega \backslash \Omega_{1}, \\
& \boldsymbol{u}(\boldsymbol{x}, t)=\overline{\boldsymbol{u}}(\boldsymbol{x}, t) \quad \forall \boldsymbol{x} \in \Gamma_{\boldsymbol{u}} .
\end{aligned}
$$

\section{Static admissibility}

$\rho(\boldsymbol{x}) \ddot{\boldsymbol{u}}(\boldsymbol{x}, t)=\operatorname{div} \boldsymbol{\sigma}(\boldsymbol{x}, t)+\int_{\mathcal{H}_{\delta}(\boldsymbol{x})} \boldsymbol{f}\left(\boldsymbol{x}^{\prime}, \boldsymbol{x}, t\right) d V_{\boldsymbol{x}^{\prime}}+\boldsymbol{b}(\boldsymbol{x}, t) \quad \forall \boldsymbol{x} \in \Omega$,

$\boldsymbol{\sigma}(\boldsymbol{x}, t) \boldsymbol{n}(\boldsymbol{x}, t)=\overline{\boldsymbol{T}}(\boldsymbol{x}, t) \quad \forall \boldsymbol{x} \in \Gamma_{\boldsymbol{T}}$.

\section{Constitutive equations}

$$
\begin{aligned}
& \sigma(x, t)=\boldsymbol{E}(\boldsymbol{x}): \boldsymbol{\varepsilon}(\boldsymbol{x}, t) \quad \forall \boldsymbol{x} \in \Omega \backslash \Omega_{2}, \\
& \boldsymbol{f}\left(\boldsymbol{x}^{\prime}, \boldsymbol{x}, t\right)=\frac{1}{2}\left(\boldsymbol{C}(\boldsymbol{x}, \boldsymbol{\xi})+\boldsymbol{C}\left(\boldsymbol{x}^{\prime}, \boldsymbol{\xi}\right)\right) \eta\left(\boldsymbol{x}^{\prime}, \boldsymbol{x}, t\right) \quad \forall \boldsymbol{x}, \boldsymbol{x}^{\prime} \in \Omega \backslash \Omega_{1},
\end{aligned}
$$

where $\varepsilon$ and $\sigma$ are the small strain and Cauchy stress tensor, respectively. $\boldsymbol{\eta}$ is the vector of relative displacements. In Eq.
174 175 176 177 178 179 180 181 182 183 184 185 186 187 188 
(11), $\boldsymbol{E}(\boldsymbol{x})$ is the stiffness tensor of the classical continuum model at the point $\boldsymbol{x}$. In Eq. (12), $\boldsymbol{C}(\boldsymbol{x}, \boldsymbol{\xi})$ is the micromodulus tensor redefined as follows [23]:

$\boldsymbol{C}(\boldsymbol{x}, \boldsymbol{\xi})=\alpha(\boldsymbol{x}) c^{0}(\boldsymbol{\xi}) \boldsymbol{\xi} \otimes \boldsymbol{\xi}$,

where $\alpha(\boldsymbol{x})$ is a Morphing function $(0 \leq \alpha(\boldsymbol{x}) \leq 1, \forall \boldsymbol{x} \in$ $\Omega)$, and $c^{0}(\xi)$ is a scalar-valued function as in the references. Comparing Eq. (5) with Eq. (13), we know that $c(\boldsymbol{x}, \boldsymbol{\xi})=$ $\alpha(\boldsymbol{x}) c^{0}(\boldsymbol{\xi}) . \boldsymbol{C}\left(\boldsymbol{x}^{\prime}, \boldsymbol{\xi}\right)$ has the same definition with respect to $\boldsymbol{x}^{\prime}$ and $\boldsymbol{\xi}$. Note that both the stiffness $\boldsymbol{E}$ and micromodulus $\boldsymbol{C}$ as material properties are independent of time $t$.

Taking account of Eqs. (9), (11), (12) and (13), we find that both functions $\alpha(\boldsymbol{x})$ and $\boldsymbol{E}(\boldsymbol{x})$ can drive the morphing between the classical continuum model and the peridynamic model at a given time $t$ over an internal subdomain $\Omega_{0}$ in which we can avoid an incomplete neighborhood of points when the peridynamic model is used near the boundary of $\Omega$. This subdomain, $\Omega_{0}$, is exactly defined as $\Omega_{0}=\{x \in \Omega$ : $\left.\mathcal{H}_{\delta}(\boldsymbol{x}) \subset \Omega\right\}[1]$. Now let us consider the exact model at a point $\boldsymbol{x}\left(\boldsymbol{x} \in \Omega_{0}\right)$ and any time $t[15,22,23]$ :

- If and only if $\boldsymbol{E}(\boldsymbol{x})=\boldsymbol{E}^{0}$ and $\alpha\left(\boldsymbol{x}^{\prime}\right)=0, \forall \boldsymbol{x}^{\prime} \in \mathcal{H}_{\delta}(\boldsymbol{x})$, then the model at this point, $\boldsymbol{x}$, is restricted to the classical continuum model. The strain energy density can be written as

$W(\boldsymbol{x}, t)=\frac{1}{2} \boldsymbol{\varepsilon}(\boldsymbol{x}, t): \boldsymbol{E}^{0}: \boldsymbol{\varepsilon}(\boldsymbol{x}, t)$.

- If and only if $\boldsymbol{E}(\boldsymbol{x})=\mathbf{0}$ and $\alpha\left(\boldsymbol{x}^{\prime}\right)=1, \forall \boldsymbol{x}^{\prime} \in \mathcal{H}_{\delta}(\boldsymbol{x})$, then the model at this point, $\boldsymbol{x}$, is restricted to the peridynamic model. The elastic energy density at this point can be written as (see [14,23])

$$
W(\boldsymbol{x}, t)=\frac{1}{4} \int_{\mathcal{H}_{\delta}(\boldsymbol{x})} c^{0}(\boldsymbol{\xi})\left(\boldsymbol{\xi} \cdot \boldsymbol{\eta}\left(\boldsymbol{x}^{\prime}, \boldsymbol{x}, t\right)\right)^{2} d V_{\boldsymbol{x}^{\prime}}
$$

- If and only if $\boldsymbol{E}(\boldsymbol{x}) \neq \mathbf{0}$ and $\exists \boldsymbol{x}^{\prime} \in \mathcal{H}_{\delta}(\boldsymbol{x})$, such that $0<\alpha\left(\boldsymbol{x}^{\prime}\right)<1$, then we can say the model at this point, $\boldsymbol{x}$, is a hybrid model. The elastic energy density at this point can be written as (see [15])

$$
\begin{aligned}
W(\boldsymbol{x}, t)= & \frac{1}{2} \boldsymbol{\varepsilon}(\boldsymbol{x}, t): \boldsymbol{E}(\boldsymbol{x}): \boldsymbol{\varepsilon}(\boldsymbol{x}, t) \\
& +\frac{1}{4} \int_{\mathcal{H}_{\delta}(\boldsymbol{x})} c^{0}(\boldsymbol{\xi}) \frac{\alpha(\boldsymbol{x})+\alpha\left(\boldsymbol{x}^{\prime}\right)}{2}\left(\boldsymbol{\xi} \cdot \boldsymbol{\eta}\left(\boldsymbol{x}^{\prime}, \boldsymbol{x}, t\right)\right)^{2} d V_{\boldsymbol{x}^{\prime}}
\end{aligned}
$$

If the material properties in $\Omega$ is homogeneous and the body, $\Omega$, is under homogeneous deformation at any time $t$, the elastic energy density should be independent of the Morphing function $\alpha$. This means that the elastic energy density is the same at some point $\boldsymbol{x}$ whatever the model is. Thus, (14) is equal to (15), i.e.,

$$
\begin{aligned}
& \frac{1}{2} \boldsymbol{\varepsilon}(\boldsymbol{x}, t): \boldsymbol{E}^{0}: \boldsymbol{\varepsilon}(\boldsymbol{x}, t) \\
& \quad=\frac{1}{4} \int_{\mathcal{H}_{\delta}(\boldsymbol{x})} c^{0}(\boldsymbol{\xi})\left(\boldsymbol{\xi} \cdot \eta\left(\boldsymbol{x}^{\prime}, \boldsymbol{x}, t\right)\right)^{2} d V_{\boldsymbol{x}^{\prime}}
\end{aligned}
$$

Let us consider an infinitesimal homogeneous transformation over the small neighborhood of point $\boldsymbol{x}$ such that

$$
\begin{aligned}
\boldsymbol{\varepsilon}\left(\boldsymbol{x}^{\prime}, t\right) \backsim \boldsymbol{\varepsilon}(\boldsymbol{x}, t) & =\overline{\boldsymbol{\varepsilon}}(t) \text { and } \boldsymbol{\eta}\left(\boldsymbol{x}^{\prime}, \boldsymbol{x}, t\right) \\
& =\overline{\boldsymbol{\varepsilon}}(t) \cdot \boldsymbol{\xi}, \quad \forall \boldsymbol{x}^{\prime} \in \mathcal{H}_{\delta}(\boldsymbol{x}) .
\end{aligned}
$$

Based on (18), (17) yields

$\boldsymbol{E}^{0}=\frac{1}{2} \int_{\mathcal{H}_{\delta}(\boldsymbol{x})} c^{0}(\boldsymbol{\xi}) \xi \otimes \xi \otimes \xi \otimes \xi d V_{\boldsymbol{x}^{\prime}}$

Similarly, an equivalence also exists between (14) and (16), and considering (18) and (19), we have

$\boldsymbol{E}(\boldsymbol{x})=\boldsymbol{E}^{0}-\frac{1}{2} \int_{\mathcal{H}_{\delta}(\boldsymbol{x})} c^{0}(\boldsymbol{\xi}) \frac{\alpha(\boldsymbol{x})+\alpha\left(\boldsymbol{x}^{\prime}\right)}{2} \boldsymbol{\xi} \otimes \boldsymbol{\xi} \otimes \boldsymbol{\xi} \otimes \boldsymbol{\xi} d V_{\boldsymbol{x}^{\prime}}$

The resulting Eqs. (19) and (20) illustrate the stiffness tensor is independent of the time. Finally, we obtain the hybrid local/nonlocal continuum model (i.e., Eqs. 6-13 and Eq. 20) for dynamic problems.

Remark Weighting functions are commonly chosen to be used in coupling techniques [14,15,24,25]. These weighting functions can usually be different shapes such as a linear function, a cubic function, or a Heaviside function. In the Morphing method, the coupling errors (i.e., the so-called ghost forces) with the cubic weighting function is smaller than the errors with the linear or Heaviside function; this has been analytically proved in [15] and also in this paper. Moreover, note that no additional parameters, such as Lagrange multipliers, are induced by the Morphing method.

\section{Theoretical analysis}

\subsection{Harmonic wave solution in hybrid local/nonlocal continuum model}

Let us consider a homogeneous body, $\Omega$, which is composed of the linearly elastic material described above. In order to facilitate theoretical analysis without loss of generality, we only consider isotropic materials in this section, i.e., the coefficient function of the micromodulus tensor $c^{0}(\xi)=c^{0}(|\xi|)$. 
In the internal subregion $\Omega_{0}$ of $\Omega$, we then investigate the propagation of plane harmonic waves:

$\boldsymbol{u}(\boldsymbol{x}, t)=\boldsymbol{a} e^{i \kappa(\boldsymbol{g} \cdot \boldsymbol{x}-v t)}$,

where $\boldsymbol{a}$ is the amplitude vector, $i=\sqrt{-1}, \kappa$ is the positive wavenumber, $\boldsymbol{g}=\left[g_{1}, g_{2}, g_{3}\right]$ is a unit vector that gives the direction of propagation, $v$ is the wave speed and $v=\omega / \kappa$, where $\omega$ is the angular frequency. The components of $\boldsymbol{a}$ may be complex, but only the real part of the right hand side of Eq. (21) represents a physical displacement [1].

Substituting Eq. (21) into the left-hand side of Eq. (9) yields

$\rho \ddot{\boldsymbol{u}}(\boldsymbol{x}, t)=-\rho \kappa^{2} v^{2} e^{i \kappa(\boldsymbol{g} \cdot \boldsymbol{x}-v t)} \boldsymbol{a}$,

where the density $\rho$ is assumed to be constant. We then substitute Eqs. (6) and (11) into the first term of the right-hand side of Eq. (9), yielding

$\operatorname{div} \boldsymbol{\sigma}(\boldsymbol{x}, t)=\nabla \cdot\left\{\boldsymbol{E}(\boldsymbol{x}): \frac{1}{2}\left(\nabla \boldsymbol{u}(\boldsymbol{x}, t)+(\nabla \boldsymbol{u})^{\mathrm{T}}(\boldsymbol{x}, t)\right)\right\}$.

After that, substituting Eq. (21) into Eq. (23) and considering the symmetry of stiffness tensor $\boldsymbol{E}(\boldsymbol{x})$, Eq. (23) can be rewritten in the form of a matrix as follows

$$
\begin{aligned}
{[\operatorname{div} \boldsymbol{\sigma}]=} & \left\{-\kappa^{2} e^{i \kappa(\boldsymbol{g} \cdot \boldsymbol{x}-v t)} \boldsymbol{B}^{\mathrm{T}}(\boldsymbol{g})[\boldsymbol{E}(\boldsymbol{x})] \boldsymbol{B}(\boldsymbol{g})\right. \\
& \left.+i \kappa e^{i \kappa(\boldsymbol{g} \cdot \boldsymbol{x}-v t)} \boldsymbol{H}^{\mathrm{T}}[\boldsymbol{E}(\boldsymbol{x})] \boldsymbol{B}(\boldsymbol{g})\right\} \boldsymbol{a},
\end{aligned}
$$

where $[\operatorname{div} \sigma]$ is a vector defined by the divergence of the stress tensor, $[\boldsymbol{E}(\boldsymbol{x})]$ denotes a stiffness matrix corresponding to the fourth-order tensor $\boldsymbol{E}(\boldsymbol{x})$ at the point $\boldsymbol{x}, \boldsymbol{B}$ and $\boldsymbol{H}$ are matrices of direction vector and differential operator, respectively, defined as follows:

$302 \quad \boldsymbol{B}(\boldsymbol{g})=\left[\begin{array}{ccc}g_{1} & 0 & 0 \\ 0 & g_{2} & 0 \\ 0 & 0 & g_{3} \\ g_{2} & g_{1} & 0 \\ 0 & g_{3} & g_{2} \\ g_{3} & 0 & g_{1}\end{array}\right], \quad \boldsymbol{H}=\left[\begin{array}{ccc}\frac{\partial}{\partial x_{1}} & 0 & 0 \\ 0 & \frac{\partial}{\partial x_{2}} & 0 \\ 0 & 0 & \frac{\partial}{\partial x_{3}} \\ \frac{\partial}{\partial x_{2}} & \frac{\partial}{\partial x_{1}} & 0 \\ 0 & \frac{\partial}{\partial x_{3}} & \frac{\partial}{\partial x_{2}} \\ \frac{\partial}{\partial x_{3}} & 0 & \frac{\partial}{\partial x_{1}}\end{array}\right]$.

Substituting Eqs. (7), (12), (13), and (21) into the second term on the right-hand side of Eq. (9) yields

$$
\begin{aligned}
& \int_{\mathcal{H}_{\delta}(\boldsymbol{x})} \boldsymbol{f}\left(\boldsymbol{x}^{\prime}, \boldsymbol{x}, t\right) d V_{\boldsymbol{x}^{\prime}} \\
& =\left[\int_{\mathcal{H}_{\delta}(\boldsymbol{x})} c^{0}(|\boldsymbol{\xi}|) \frac{\alpha(\boldsymbol{x})+\alpha\left(\boldsymbol{x}^{\prime}\right)}{2}\right.
\end{aligned}
$$

$$
\left.\left(e^{i \kappa\left(\boldsymbol{g} \cdot \boldsymbol{x}^{\prime}-v t\right)}-e^{i \kappa(\boldsymbol{g} \cdot \boldsymbol{x}-v t)}\right) \xi \otimes \boldsymbol{\xi} d V_{\boldsymbol{x}^{\prime}}\right] \boldsymbol{a} .
$$

Now, we substitute Eqs. (22), (24) and (26) into Eq. (9). Then, the new equation can be shown as follows (ignoring the body force):

$$
\begin{aligned}
& -\rho \kappa^{2} v^{2} e^{i \kappa(\boldsymbol{g} \cdot \boldsymbol{x}-v t)} \boldsymbol{a}=\left\{-\kappa^{2} e^{i \kappa(\boldsymbol{g} \cdot \boldsymbol{x}-v t)} \boldsymbol{B}^{\mathrm{T}}(\boldsymbol{g})[\boldsymbol{E}(\boldsymbol{x})] \boldsymbol{B}(\boldsymbol{g})\right. \\
& \left.+i \kappa e^{i \kappa(\boldsymbol{g} \cdot \boldsymbol{x}-v t)} \boldsymbol{H}^{\mathrm{T}}[\boldsymbol{E}(\boldsymbol{x})] \boldsymbol{B}(\boldsymbol{g})\right\} \boldsymbol{a} \\
& +\left[\int _ { \mathcal { H } _ { \delta } ( \boldsymbol { x } ) } c ^ { 0 } ( | \boldsymbol { \xi } | ) \frac { \alpha ( \boldsymbol { x } ) + \alpha ( \boldsymbol { x } ^ { \prime } ) } { 2 } \left(e^{i \kappa\left(\boldsymbol{g} \cdot \boldsymbol{x}^{\prime}-v t\right)}\right.\right. \\
& \left.\left.-e^{i \kappa(\boldsymbol{g} \cdot \boldsymbol{x}-v t)}\right) \xi \otimes \xi d V_{\boldsymbol{x}^{\prime}}\right] \boldsymbol{a}
\end{aligned}
$$

308 309 310

$$
311
$$

After that, the real part of Eq. (27) yields the following eigenvalue problem, which involves the equation of wave speed.

$\rho v^{2} \boldsymbol{a}=\boldsymbol{D}(\boldsymbol{g}, \boldsymbol{x}, \kappa) \boldsymbol{a}$,

where $\boldsymbol{D}$ is a matrix given by

$$
\begin{aligned}
& \boldsymbol{D}(\boldsymbol{g}, \boldsymbol{x}, \kappa)=\boldsymbol{B}^{\mathrm{T}}(\boldsymbol{g})[\boldsymbol{E}(\boldsymbol{x})] \boldsymbol{B}(\boldsymbol{g}) \\
& +\left[\frac{1}{\kappa^{2}} \int_{\mathcal{H}_{\delta}(\boldsymbol{x})} c^{0}(|\boldsymbol{\xi}|) \frac{\alpha(\boldsymbol{x})+\alpha\left(\boldsymbol{x}^{\prime}\right)}{2}(1-\cos (\kappa \boldsymbol{g} \cdot \boldsymbol{\xi})) \boldsymbol{\xi} \otimes \boldsymbol{\xi} d V_{\boldsymbol{x}^{\prime}}\right],
\end{aligned}
$$

where we used the Euler's formula. $e^{i \kappa g \cdot \xi}=\cos (\kappa g \cdot \xi)+$ $i \sin (\kappa \boldsymbol{g} \cdot \boldsymbol{\xi})$, and $\boldsymbol{E}(\boldsymbol{x})$ can be calculated by Eq. (20). Note that the imaginary part of Eq. (27) has been removed from Eq. (29), although its coefficient does not vanish for any nonzero vector $\boldsymbol{a}$ when the stiffness tensor $\boldsymbol{E}$ isn't constant. Here, this imaginary part can be ignored because it doesn't affect the solution of the speed wave. Indeed, the imaginary part of Eq. (27) is derived from the expression of displacement in Eq. (21), which is written as a complex exponential function, $e^{i \kappa(\boldsymbol{g} \cdot \boldsymbol{x}-v t)}$, only for the sake of calculation.

\subsection{Dispersion analysis of wave propagation}

So far, we have derived the above eigenvalue problem, i.e., Eq. (28), which provides us with the speed and amplitude of plane harmonic waves. Now, we use the strategy proposed in [1] to estimate the wave speeds using Eq. (28). The following Sects. 4.2.1 and 4.2.2 reproduce these formulations to provide the complete statement in this paper.

For a plane wave in the linearly elastic, isotropic material, we fix the direction of wave propagation, $g$, by defining an orthonormal basis $\left\{\boldsymbol{e}_{1}, \boldsymbol{e}_{2}, \boldsymbol{e}_{3}\right\}$, such that $\boldsymbol{e}_{1}=\boldsymbol{g}$. We then derive the expressions of wave speed by considering the exact form of Eq. (29) at a point, $\boldsymbol{x}\left(\boldsymbol{x} \in \Omega_{0}\right)$. We can see below that the form of Eq. (29) is determined by the values of both $\alpha(\boldsymbol{x})$ and $\boldsymbol{E}(\boldsymbol{x})$. 
${ }_{362} v_{c, d}^{2}=\frac{\lambda+2 \mu}{\rho}, \quad v_{c, s}^{2}=\frac{\mu}{\rho}$,

$\rho v_{c}^{2} \boldsymbol{a}=\boldsymbol{D}_{c}(\boldsymbol{g}) \boldsymbol{a}$, propagation, i.e., $\boldsymbol{g}=\boldsymbol{e}_{1}$,

$\boldsymbol{D}_{c}\left(\boldsymbol{e}_{1}\right)=\left[\begin{array}{ccc}\lambda+2 \mu & 0 & 0 \\ 0 & \mu & 0 \\ 0 & 0 & \mu\end{array}\right]$ by this paper.

$\rho v_{p}^{2} \boldsymbol{a}=\boldsymbol{D}_{p}(\boldsymbol{g}, \kappa) \boldsymbol{a}$,

\subsubsection{In the domain of classical continuum model}

Let us consider that a point $\boldsymbol{x}$ is restricted to the classical continuum model, i.e., $x \in \Omega_{1}$. As we mentioned above, it is true if and only if $\boldsymbol{E}(\boldsymbol{x})=\boldsymbol{E}^{0}$ and $\alpha\left(\boldsymbol{x}^{\prime}\right)=0, \forall \boldsymbol{x}^{\prime} \in \mathcal{H}_{\delta}(\boldsymbol{x})$. In this case, the expression of $\boldsymbol{D}$ yields

$\boldsymbol{D}_{c}(\boldsymbol{g})=\boldsymbol{B}^{\mathrm{T}}(\boldsymbol{g})\left[\boldsymbol{E}^{0}\right] \boldsymbol{B}(\boldsymbol{g})$.

Equation (28) can be recast as

where $v_{c}$ denotes the wave speeds in the domain of the classical continuum model. For the fixed direction of wave

where $\lambda$ is the first Lamé modulus and $\mu$ is the shear modulus. According to Eq. (31), the $\rho v_{c}^{2}$ are the eigenvalues of $\boldsymbol{D}_{c}$, and the wave speeds for dilatational and shear waves are given

where the subscripts $d$ and $s$ denote their dilatational and shear components, respectively. Both speeds in Eq. (33) are independent of frequency denoted by the wavenumber $\kappa$ in

\subsubsection{In the domain of peridynamic model}

In another case, a point $\boldsymbol{x}$ is strictly belonging to the peridynamic domain, $\Omega_{2}$, where if and only if $\boldsymbol{E}(\boldsymbol{x})=\mathbf{0}$ and $\alpha\left(\boldsymbol{x}^{\prime}\right)=1, \forall \boldsymbol{x}^{\prime} \in \mathcal{H}_{\delta}(\boldsymbol{x})$. Then, the expression of $\boldsymbol{D}$ yields

$\boldsymbol{D}_{p}(\boldsymbol{g}, \kappa)=\left[\frac{1}{\kappa^{2}} \int_{\mathcal{H}_{\delta}(\mathbf{0})} c^{0}(|\boldsymbol{\xi}|)(1-\cos (\kappa \boldsymbol{g} \cdot \boldsymbol{\xi})) \boldsymbol{\xi} \otimes \boldsymbol{\xi} d V_{\boldsymbol{\xi}}\right]$

Equation (28) can be recast as

where $v_{p}$ denotes the wave speeds in the domain of the peridynamic model. We estimate the integration of Eq. (34) by using a spherical coordinate system [17]. The details of derivation can be found in [1]. According to Eq. (35), the wave speeds are the same as those calculated by Eq. (33) when $\kappa \rightarrow 0$, which means the wave speeds are almost constant when the wave with smaller wavenumber goes through the peridynamic domain.

On the other hand, the speeds of a wave with larger wavenumber in the peridynamic domain are completely different from those in the classical continuum mechanics domain. As long as $\kappa \rightarrow \infty$, the wave speeds derived from Eqs. (34) and (35) yield

$v_{p, d}^{2}=v_{p, s}^{2}=\frac{4 \pi}{3 \rho \kappa^{2}} \int_{0}^{\delta} c^{0}(r) r^{4} d r$,

where the subscripts $d$ and $s$ denote the dilatational and shear components, respectively. Both speeds depend on the wavenumber $\kappa$ [1]. Furthermore, Eq. (36) shows that the wave speeds have the same value in both dilatational and shear waves, which is different from the smaller wavenumber case estimated by Eq. (33) where the dilatational and shear waves have different speeds of propagation.

\subsubsection{In the domain of hybrid local/nonlocal continuum model}

Both the classical continuum model and the peridynamic model coexist in the hybrid domain, $\Omega_{m}$, in which a point $\boldsymbol{x}$ satisfies $\boldsymbol{E}(\boldsymbol{x}) \neq \mathbf{0}$ and $\exists \boldsymbol{x}^{\prime} \in \mathcal{H}_{\delta}(\boldsymbol{x})$, such that $0<\alpha\left(\boldsymbol{x}^{\prime}\right)<$ 1. Then, the wave speeds in the hybrid domain can be accurately calculated by Eq. (28). Additionally, we will discuss the wave speeds of an ideal case in which no false wave is reflected in the hybrid domain.

We apply a partition-of-unity concept using the Morphing function in the hybrid domain. The left-hand side of Eq. (28) yields

$\rho v^{2} \boldsymbol{a}=(1-\alpha(\boldsymbol{x})) \rho v^{2} \boldsymbol{a}+\alpha(\boldsymbol{x}) \rho v^{2} \boldsymbol{a}$

We know that Eq. (37) still holds in both the classicial continuum domain where $\alpha(\boldsymbol{x})=0$ and the peridynamic domain where $\alpha(\boldsymbol{x})=1$. Consequently, Eq. (37) can be recast as

$$
\begin{aligned}
\rho v^{2} \boldsymbol{a} & =(1-\alpha(\boldsymbol{x})) \rho v_{c}^{2} \boldsymbol{a}+\alpha(\boldsymbol{x}) \rho v_{p}^{2} \boldsymbol{a} \\
& =(1-\alpha(\boldsymbol{x})) \boldsymbol{D}_{c}(\boldsymbol{g}) \boldsymbol{a}+\alpha(\boldsymbol{x}) \boldsymbol{D}_{p}(\boldsymbol{g}, \kappa) \boldsymbol{a} \\
& =\boldsymbol{D}_{h}(\boldsymbol{g}, \boldsymbol{x}, \kappa) \boldsymbol{a},
\end{aligned}
$$

where

$$
\begin{aligned}
\boldsymbol{D}_{h}(\boldsymbol{g}, \boldsymbol{x}, \kappa)= & (1-\alpha(\boldsymbol{x})) \boldsymbol{B}^{\mathrm{T}}(\boldsymbol{g})\left[\boldsymbol{E}^{0}\right] \boldsymbol{B}(\boldsymbol{g}) \\
& +\frac{\alpha(\boldsymbol{x})}{\kappa^{2}}\left[\int_{\mathcal{H}_{\delta}(\boldsymbol{0})} c^{0}(|\boldsymbol{\xi}|)(1-\cos (\kappa \boldsymbol{g} \cdot \boldsymbol{\xi})) \xi \otimes \xi d V_{\boldsymbol{\xi}}\right] .
\end{aligned}
$$

The second step of Eq. (38) is yielded by substituting Eqs. (31) and (35). In fact, the eigenvalue Eq. (38) demonstrates 
both local and nonlocal continuum models are assembled by a perfect partition of unity using a Morphing function. It makes an ideal way to couple classical continuum mechanics with peridynamics in the hybrid domain, by which the incoming waves will smoothly and completely pass through the hybrid domain from the classical continuum domain into the peridynamic domain, and vice versa. Consequently, according to Eqs. (38) and (39), we can obtain the same speed as Eq. (33) when $\kappa \rightarrow 0$. On the other hand, for $\kappa \rightarrow \infty$, the wave speeds yield as follows:

$$
\begin{aligned}
& v_{d}^{2}=(1-\alpha(\boldsymbol{x})) \frac{\lambda+2 \mu}{\rho}+\alpha(\boldsymbol{x}) \frac{4 \pi}{3 \rho \kappa^{2}} \int_{0}^{\delta} c^{0}(r) r^{4} d r \\
& v_{s}^{2}=(1-\alpha(\boldsymbol{x})) \frac{\mu}{\rho}+\alpha(\boldsymbol{x}) \frac{4 \pi}{3 \rho \kappa^{2}} \int_{0}^{\delta} c^{0}(r) r^{4} d r .
\end{aligned}
$$

From Eq. (40), we can see that when $\alpha(\boldsymbol{x}) \rightarrow 0$, the wave speeds are approximately independent of the wavenumber, while the wave trends to dispersion when $\alpha(\boldsymbol{x}) \rightarrow 1$.

Equation (38) or expression (40) represent an ideal wave transmission process in the hybrid domain, where the Morphing function $\alpha$ changes in the range $[0,1]$, that is to say, no false wave reflection occurs, which also indicates that non-physical effects (i.e., ghost forces) do not exist in this hybrid mode. Therefore, we can apply Eq. (38) as a reference case (perfect coupling) and compare it with Eq. (28) to estimate ghost force and its work. We will discuss this further in Sect. 4.4. Before we discuss ghost force and its work, we plot and compare the ideal solutions with numerical results calculated by Eq. (28) in the following section, from which we can achieve an intuitive grasp of wave dispersion and the morphing effect.

Remark Equation (38) is actually an approximation of Eq. (28); we can obtain Eq. (38) by replacing the function $\alpha(\boldsymbol{x}+$ $\boldsymbol{\xi})$ with its first-order Taylor approximation at $\boldsymbol{x} \in \Omega_{m_{0}}$ where $\Omega_{m_{0}}=\left\{\boldsymbol{x} \in \Omega_{m}: \mathcal{H}_{\delta}(\boldsymbol{x}) \subset \Omega_{m}\right\}$ (i.e., $\alpha(\boldsymbol{x}+\boldsymbol{\xi})=\alpha(\boldsymbol{x})+$ $\left.\frac{\partial \alpha(\boldsymbol{x})}{\partial \boldsymbol{x}} \cdot \boldsymbol{\xi}\right)$ in the expression of $\boldsymbol{D}$ (i.e., Eq. 29), as long as the Morphing function $\alpha$ is piecewise continuously differentiable over the hybrid domain. However, this does not prevent Eq. (38) from being an ideal coupling to serve as a reference configuration for the hybrid model. Note that in Sect. 4.4, we will apply the concept of ideal coupling as a reference, rather than an approximate solution, to estimate ghost force and the work it does.

\subsection{Numerical analysis of wave dispersion relations}

In this section, we display the dispersion relation of the wave and compare the angular frequency changes of the reference solution and the numerical solution on the hybrid local/nonlocal domain. A dispersion relation describes the effect of wave dispersion on the wave propagation within a medium. Usually, the dispersion relation is defined by the angular frequency $\omega(\kappa)$, which is a function of wavenumber $\kappa$. Furthermore, this function $\omega(\kappa)$ can be written as $\omega(\kappa)=v(\kappa) \kappa$. Here, the wavenumber, $\kappa$, is the spatial frequency of a wave, measured in radians per unit distance. Since the frequency of waves in a peridynamic model is affected by the peridynamic horizon $\delta$, we redefined the wavenumber as $q=\kappa /(2 \pi / \delta)$, which is the number of wavelengths per horizon. Henceforth, $q$ is called the peridynamic wavenumber. Consequently, an expression of the reference angular frequency can be written according to the wave speeds in the ideal hybrid model for $\kappa \rightarrow \infty$ (i.e., Eq. 40) as follows:

$$
\begin{aligned}
\omega_{d}^{2} & =(1-\alpha(\boldsymbol{x})) \frac{4 \pi^{2}(\lambda+2 \mu)}{\rho \delta^{2}} q^{2}+\alpha(\boldsymbol{x}) \frac{4 \pi}{3 \rho} \int_{0}^{\delta} c^{0}(r) r^{4} d r \\
\omega_{s}^{2} & =(1-\alpha(\boldsymbol{x})) \frac{4 \pi^{2} \mu}{\rho \delta^{2}} q^{2}+\alpha(\boldsymbol{x}) \frac{4 \pi}{3 \rho} \int_{0}^{\delta} c^{0}(r) r^{4} d r
\end{aligned}
$$

where the subscript $d$ and $s$ denote the dilatational and shear waves, respectively. This hybrid expression of angular frequency (i.e., Eq. 41) are still true for a purely peridynamic model when $\alpha(\boldsymbol{x})=1$ and for a purely classical continuum model when $\alpha(\boldsymbol{x})=0$. It does not mean, however, that the dispersion relations of the classical continuum model depend on $\delta$, but because the following equation holds:

$$
\frac{4 \pi^{2}(\lambda+2 \mu)}{\rho \delta^{2}} q^{2}=\frac{(\lambda+2 \mu)}{\rho} \kappa^{2},
$$

due to the wavenumber $q$ as defined by $\kappa /(2 \pi / \delta)$.

Then, we plot the angular frequencies $\omega$ of dilatational and shear waves as functions of wavenumber $q$ and reference location $x$ along the direction of wave propagation, $\boldsymbol{g}$. Here we consider the simple case where the Morphing function $\alpha$ is a cubic function on the reference location $x$ in the domain, $\Omega_{m_{0}}$. In addition to plotting the reference dispersion relations defined by Eq. (41), we also plot the numerical solutions of angular frequencies $\omega$ based on the basic eigenvalue equations, i.e., Eqs. (28) and (29). Then, we compare the numerical solutions of the dispersion surfaces on the boundary of the calculation domain with the reference solution defined by Eq. (41).

Let the density for the material be $\rho=8000 \mathrm{~kg} / \mathrm{m}^{3}$, and the Young's modulus $E$ of this material be $200 \mathrm{GPa}$ [1]. Let $\Omega$ start from the origin and the length along the propagation direction, $\boldsymbol{g}$, be $2 \mathrm{~m}$, and the domain, $\Omega_{m_{0}}$, be $[0.6,1.4] \mathrm{m}$. $L$ denotes the length of $\Omega_{m_{0}}$. A micro-modulus, $c^{0}(r)$, in power function is assumed to be $c^{0}(r)=9 E /\left(\pi \delta^{3} r^{4}\right)$, where the horizon $\delta=0.2 \mathrm{~m}$. For the linear elastic, isotropic material under homogeneously unidirectional deformations, we can obtain the equivalent Lamé parameters, $\lambda=\mu=2 E / 5$; refer to [1]. 


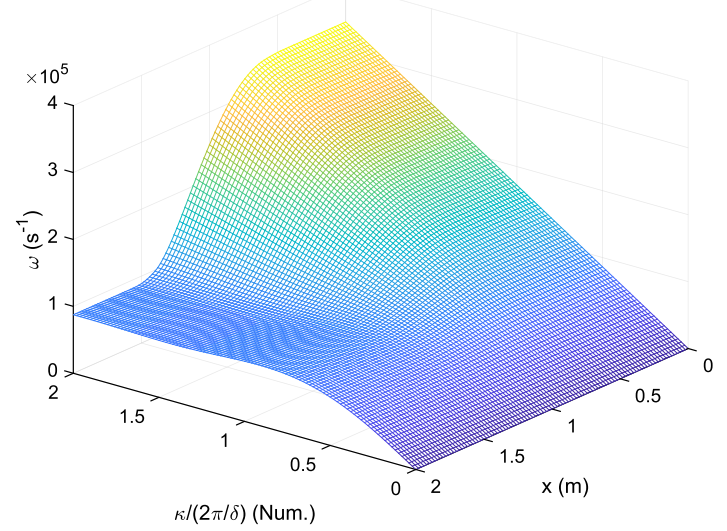

(a)

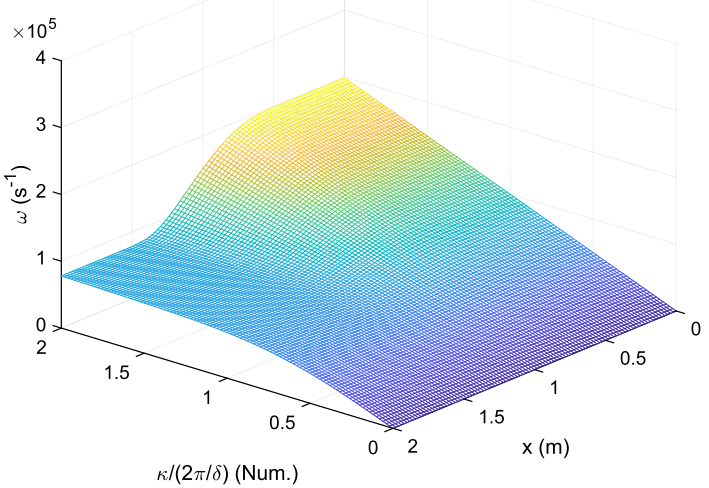

(b)

Fig. 3 The numerical dispersion surfaces: the angular frequencies of a dilatational wave and $\mathbf{b}$ shear wave, which are functions of the wavenumber in a horizon and position along the propagation direction $g$

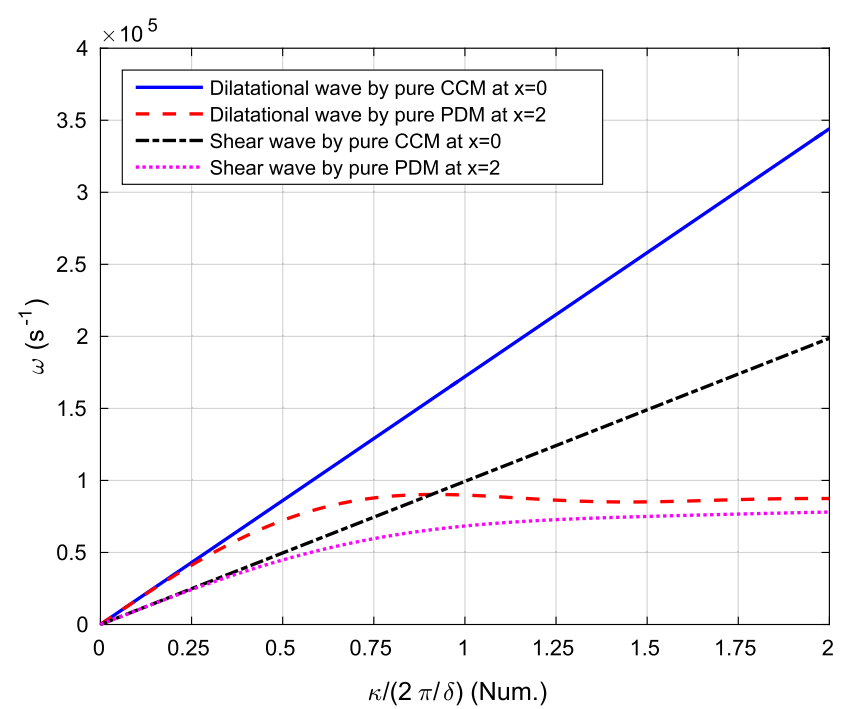

(a)

Fig. 4 a The angular frequencies of dilatational and shear waves as functions of wavenumber in a horizon are estimated by the classical continuum model (CCM) and the peridynamic model (PDM). b The

The numerical dispersion surfaces are shown in Fig. 3a for a dilatational wave and Fig. $3 \mathrm{~b}$ for a shear wave, respectively. We can see the relations between angular frequency and peridynamic wavenumber $q$ at different locations. When the value of location $x$ is near to 0 , where it is in the domain of the purely classical continuum model, the angular frequency is a linear function of wavenumber, which means that no wave dispersion occurs. When the value of location $x$ is close to 2 , where it is in the domain of purely peridynamic model, the angular frequency displays a nonlinear function of wavenumber, which means that wave dispersion occurs. Moreover, when the peridynamic wavenumber is larger than

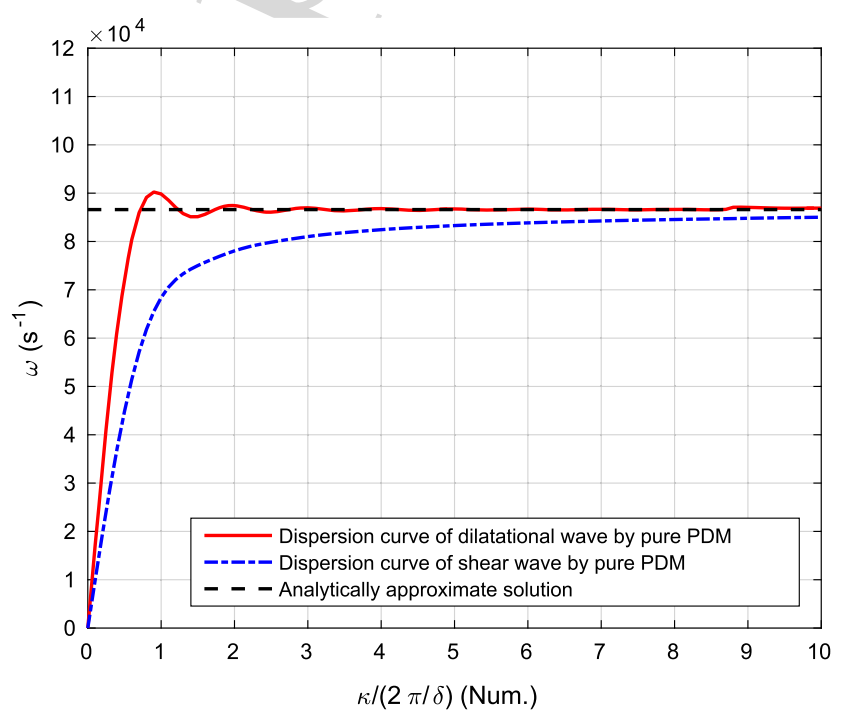

(b)

angular frequencies at $x=2$ estimated by PDM are converging to the analytical limit, i.e., Eq. (41) where $\alpha=1$, when wavenumber $q \rightarrow 10$, where $q=\kappa /(2 \pi / \delta)$

1, the angular frequency for the purely peridynamic model becomes an approximated constant, which means the speed of a wave with a larger wavenumber approaches zero in the domain of a purely peridynamic model.

Figure 4a shows the angular frequencies of dilatational and shear waves as functions of wavenumber per horizon, whose curves are plotted by numerical solutions of the purely classical continuum model and the peridynamic model. From Fig. 4a, we can see that the angular frequencies of dilatational and shear waves are linear functions of wavenumber by the classical continuum model, while the angular frequencies estimated by the peridynamic model are nonlinear functions 


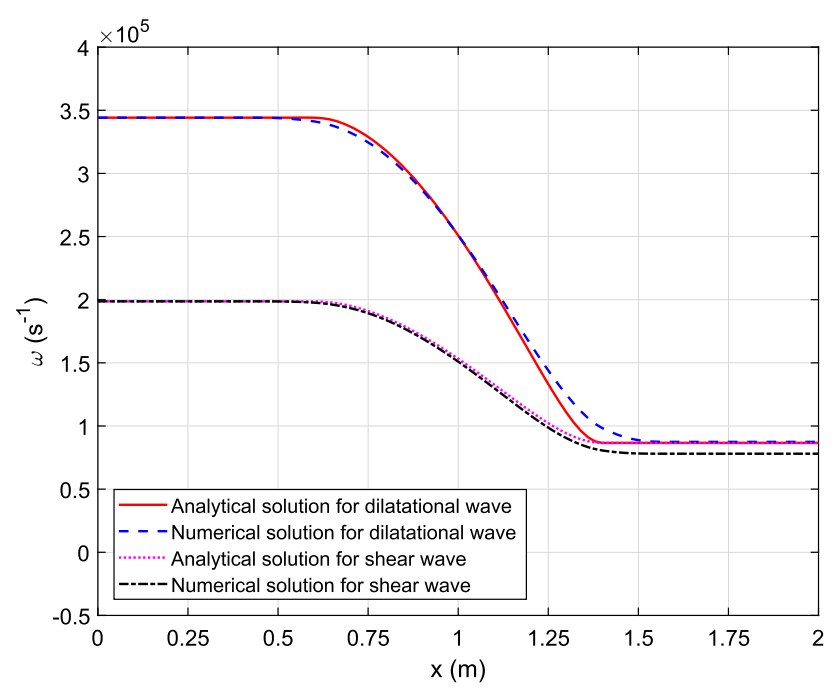

Fig. 5 The angular frequencies of waves with larger wavenumber $(q=2)$ as functions of location are compared between the numerical solutions and analytical solutions. $\Omega_{m_{0}}=[0.6,1.4] \mathrm{m}$

of wavenumber. These results confirm our statement that the nondispersive wave is predicted by the classical continuum model while the dispersive wave is predicted by the peridynamic model.

Figure $4 \mathrm{~b}$ shows that the angular frequencies of dilatational and shear waves converge to the analytical limit, i.e., Eq. (41) with $\alpha(x)=1$, when the wavenumber $q$ increases. Here, $q \in[0,10]$. By comparison, the angular frequency of the dilatational wave converges more quickly than that of the shear wave.

Figure 5 shows that the angular frequencies of dilatational and shear waves with a larger wavenumber (i.e., $q=2$ ) vary in the whole structure. According to Eq. (41), we plot the reference solutions, which represent the ideal case. On the other hand, we recalculate the speed of the harmonic wave along the propagation direction $\boldsymbol{g}$ by directly solving Eq. (28) in a numerical way. The numerical results of angular frequencies of dilatational and shear waves are also shown in Fig. 5. We found that significant errors occur near the boundary region between $\Omega_{m}$ and $\Omega_{2}$ for the dilatational wave. For the shear wave, the significant error occurs in the domain of the peridynamics model because the angular frequency doesn't converge to the analytical limit when $q=2$ (see Fig. 4b). Nevertheless, the angular frequency of the dilatational wave converges to the analytical limit when $q=2$, which can also be seen from Fig. $4 \mathrm{~b}$.

\subsection{Discussion of the coupling quality}

Based on the assumption of infinitesimal homogeneous transformation (i.e., Eq. 18) and the equivalence condition of elastic energy density (i.e., Eq. 17), the constraint between local stiffness and nonlocal micromodulus (i.e., Eq. 20) ensures that the hybrid model is energetically equivalent to the purely local continuum model or the purely peridynamic model. However, this constraint does not guarantee that perfect force equivalents can also be achieved at the same time. Consequently, the non-physical (spurious) effects arise due to approximate force equilibrium caused when addressing the local/non-local interface in the hybrid domain. These spurious effects are often referred to as "ghost forces" by the academic community. Ghost forces are a well-known concept in atomistic-to-continuum coupling [26]. In the present study, an approximate, but not precise, force equilibrium is not capable of achieving perfect wave transmission, resulting in spurious reflection. Therefore, we can make further inferences, i.e., ghost force is the root cause of the spurious reflection, and the work done by ghost force directly determines the energy of reflection. By estimating the ghost force and its work, therefore, we can understand the influence of coupling parameters on the spurious reflection. For our hybrid local/nonlocal model, the ghost forces can be evaluated by solving an inverse problem in which the wave speed is prescribed to smoothly transit without reflections in the whole domain, i.e., the speed is the solutions of the reference Eq. (38). The details of derivation about the ghost force and its work are stated as follows.

\subsubsection{Ghost forces evaluation}

Let us designate ghost forces by $\tilde{\boldsymbol{f}}_{g}(\boldsymbol{x}, t)$. As mentioned before, $\tilde{\boldsymbol{f}}_{g}(\boldsymbol{x}, t)$ are the residual of the equilibrium equation of forces under the energetically equivalent constraint. Therefore, the equilibrium equation, i.e., Eq. (9), can be recast as follows, ignoring the external body forces:

$$
\rho \ddot{\boldsymbol{u}}(\boldsymbol{x}, t)=\operatorname{div} \boldsymbol{\sigma}(\boldsymbol{x}, t)+\int_{\mathcal{H}_{\delta}(\boldsymbol{x})} \boldsymbol{f}\left(\boldsymbol{x}^{\prime}, \boldsymbol{x}, t\right) d V_{\boldsymbol{x}^{\prime}}+\tilde{\boldsymbol{f}}_{g}(\boldsymbol{x}, t) .
$$

Equation (42) is a general model prescribed to the whole computational domain including the classical continuum domain, the peridynamic domain, and the hybrid local/nonlocal 600 domain. Then, the ghost forces can be further written as follows by substituting the expression of the plane harmonic wave (i.e., Eq. (21)):

$$
\tilde{\boldsymbol{f}}_{g}(\boldsymbol{x}, t)=-\kappa^{2} e^{i \kappa(\boldsymbol{g} \cdot \boldsymbol{x}-v t)}\left(\rho v^{2} \boldsymbol{I}-\boldsymbol{D}(\boldsymbol{g}, \boldsymbol{x}, \kappa)\right) \boldsymbol{a} .
$$

To evaluate $\tilde{\boldsymbol{f}}_{g}(\boldsymbol{x}, t)$, we prescribe that the wave speeds are the solutions of the ideal hybrid model (i.e., Eq. (38)), that is, spurious reflection is absent in the whole domain. Substituting Eq. (38), Eq. (43) yields as 
$\tilde{\boldsymbol{f}}_{g}(\boldsymbol{x}, t)=-\kappa^{2} e^{i \kappa(\boldsymbol{g} \cdot \boldsymbol{x}-v t)}\left(\boldsymbol{D}_{h}(\boldsymbol{g}, \boldsymbol{x}, \kappa)-\boldsymbol{D}(\boldsymbol{g}, \boldsymbol{x}, \kappa)\right) \boldsymbol{a}$.

Let us analyze $\tilde{\boldsymbol{f}}_{g}(\boldsymbol{x}, t)$ on each domain separately as follows:

${ }_{643} \quad c^{0}=5 E^{0} / \delta^{5}$. purely nonlocal and $\alpha(\boldsymbol{x})=1$. As a result, $\tilde{\boldsymbol{f}}_{g}(\boldsymbol{x}, t)=0$ according to Eq. (44).

- In the domain of the hybrid local/nonlocal continuum model: This model is hybrid and $\exists \boldsymbol{x}^{\prime} \in \mathcal{H}_{\delta}(\boldsymbol{x})$, such that $0<\alpha\left(\boldsymbol{x}^{\prime}\right)<1$. Then, the ghost forces can be further written as follows by substituting Eqs. (20), (29), and (39):

where

\subsubsection{Ghost forces in the one-dimensional case}

In this section, we derive the precise expressions of errors. As shown in from Figs. 4 and 5, the errors of angular frequency (similar to those of speed) are related to the wavenumber $q$, the Morphing function $\alpha$, and the length $L$ of $\Omega_{m_{0}}$. We propose here to estimate the influences of these selections on the coupling errors, i.e., ghost forces $\tilde{\boldsymbol{f}}_{g}(\boldsymbol{x}, t)$.

Here, we limit ourselves to a 1D case for the sake of analysis and discussion. For a 1D bar with unit cross-sectional area, let both the Young's modulus $E^{0}$ and the corresponding peridynamic micromodulus $c^{0}$ be constant. According to Eq. (19), the following equation holds for a $1 \mathrm{D}$ homogeneous bar with unit cross-sectional area:
Substituting Eq. (46) into the 1D formulation of Eq. (45), yields:

$$
\begin{aligned}
\frac{\tilde{f}_{g}(x, t)}{\rho \tilde{\tilde{u}}_{c}(x, t)}=p(x)= & -\alpha(x)+\frac{5}{2 \delta^{5}} \int_{-\delta}^{+\delta} \frac{\alpha(x)+\alpha(x+\xi)}{2} \xi^{4} d \xi \\
& +\alpha(x) \frac{5}{\kappa^{2} \delta^{5}} \int_{-\delta}^{+\delta}[1-\cos (\kappa \xi)] \xi^{2} d \xi \\
& -\frac{5}{\kappa^{2} \delta^{5}} \int_{-\delta}^{+\delta} \frac{\alpha(x)+\alpha(x+\xi)}{2} \\
& \times[1-\cos (\kappa \xi)] \xi^{2} d \xi,
\end{aligned}
$$

$$
\begin{gathered}
\tilde{\boldsymbol{f}}_{g}(\boldsymbol{x}, t)=-\kappa^{2} e^{i \kappa(\boldsymbol{g} \cdot \boldsymbol{x}-v t)}\left(-\alpha(\boldsymbol{x}) \boldsymbol{D}_{c}(\boldsymbol{g})+\alpha(\boldsymbol{x}) \boldsymbol{D}_{p}(\boldsymbol{g}, \kappa)\right. \\
\left.+\boldsymbol{D}_{\alpha}(\boldsymbol{g}, \boldsymbol{x}, \kappa)\right) \boldsymbol{a},
\end{gathered}
$$

$$
\begin{array}{r}
\boldsymbol{D}_{\alpha}(\boldsymbol{g}, \boldsymbol{x}, \kappa)=\boldsymbol{B}^{\mathrm{T}}(\boldsymbol{g})\left[\frac{1}{2} \int_{\mathcal{H}_{\delta}(\boldsymbol{x})} c^{0}(|\boldsymbol{\xi}|) \frac{\alpha(\boldsymbol{x})+\alpha\left(\boldsymbol{x}^{\prime}\right)}{2}\right. \\
\left.\boldsymbol{\xi} \otimes \boldsymbol{\xi} \otimes \boldsymbol{\xi} \otimes \boldsymbol{\xi} d V_{\boldsymbol{x}^{\prime}}\right] \boldsymbol{B}(\boldsymbol{g}) \\
-\left[\frac{1}{\kappa^{2}} \int_{\mathcal{H}_{\delta}(\boldsymbol{x})} c^{0}(|\boldsymbol{\xi}|) \frac{\alpha(\boldsymbol{x})+\alpha\left(\boldsymbol{x}^{\prime}\right)}{2}(1\right. \\
\left.-\cos (\kappa \boldsymbol{g} \cdot \boldsymbol{\xi})) \xi \otimes \boldsymbol{\xi} d V_{\boldsymbol{x}^{\prime}}\right]
\end{array}
$$$$
I_{A}=\{\tilde{x} \mid \tilde{x}<-\delta\}, \quad I_{B}=[-\delta, 0], \quad I_{C}=[0, \delta], \quad I_{D}=[\delta, L / 2] .
$$

$-E^{0} \kappa^{2} e^{i \kappa(x-v t)} a=-\rho \kappa^{2} v_{c}^{2} e^{i \kappa(x-v t)} a=\rho \ddot{\tilde{u}}_{c}(x, t)$.

Here we discuss the ghost forces via Eq. (47). We first simplify the problem of wave propagation by focusing on half of the coupling domain (see Fig. 6). Indeed, it is sufficient to represent the ghost forces in the whole domain as these are symmetric over the coupling domain. For the sake of analytical evaluation of the ghost forces, we redefine the origin of coordinates $\tilde{x}$ to be the transition point between $\Omega_{m_{1}}$ and $\Omega_{m 0}$ (see Fig. 6), where $\Omega_{m_{0}}=\left\{\boldsymbol{x} \in \Omega_{m}: \mathcal{H}_{\delta}(\boldsymbol{x}) \subset \Omega_{m}\right\}$, $\Omega_{m_{1}}=\left\{\boldsymbol{x} \in \Omega_{m}: \mathcal{H}_{\delta}(\boldsymbol{x}) \cap \Omega_{1} \neq \emptyset\right\}$. When solving this analytical equation for a Morphing function, four intervals must be considered and defined as follows:

For clarity and without loss of generality, we limit $L \geq 2 \delta$, which can be seen from the above setting of intervals.

Based on these four intervals, we discuss two cases with different Morphing functions: the linear function and the cubic function,

Case of a linear Morphing function

$\alpha(\tilde{x})=0 \quad \forall \tilde{x} \leq 0, \quad \alpha(\tilde{x})=\tilde{x} / L \quad \forall \tilde{x} \in[0, L], \quad \alpha(\tilde{x})=1 \quad \forall \tilde{x} \geq L$.

$\frac{\tilde{f}_{g}(\tilde{x}, t)}{\rho \ddot{\tilde{u}}(\tilde{x}, t)}=\frac{\delta}{L} \Phi^{l}\left(\frac{\tilde{x}}{\delta}, \frac{\kappa \delta}{2 \pi}\right)$,

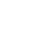

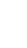

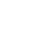




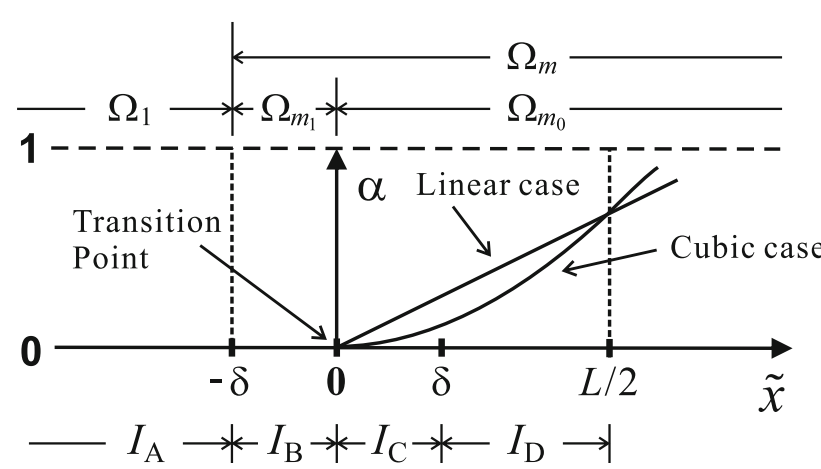

Fig. 6 A schematic of half of a coupling zone with four intervals: $I_{A}, I_{B}$, $I_{C}$ and $I_{D}$. A linear morphing function and a cubic morphing function that are used for different cases, respectively

$\Phi^{l}\left(\frac{\tilde{x}}{\delta}, \frac{\kappa \delta}{2 \pi}\right)=\left\{\begin{array}{lrl}0 & \forall \tilde{x} & \in I_{A} \\ \varphi_{1}^{l}\left(\frac{\tilde{x}}{\delta}, \frac{\kappa \delta}{2 \pi}\right) & \forall \tilde{x} \in I_{B} \\ \varphi_{1}^{l}\left(\frac{\tilde{x}}{\delta}, \frac{\kappa \delta}{2 \pi}\right)+\varphi_{2}^{l}\left(\frac{\tilde{x}}{\delta}, \frac{\kappa \delta}{2 \pi}\right) & \forall \tilde{x} \in I_{C} \\ 0 & \forall \tilde{x} \in I_{D}\end{array}\right.$

with

$$
\begin{aligned}
\varphi_{1}^{l}(y, q)= & \frac{1}{24}\left(5+6 y+y^{6}\right) \\
& -\left(\frac{5}{8(2 \pi q)^{2}}-\frac{5 \sin (2 \pi q)}{2(2 \pi q)^{3}}-\frac{15 \cos (2 \pi q)}{2(2 \pi q)^{4}}\right. \\
& \left.+\frac{15 \sin (2 \pi q)}{(2 \pi q)^{5}}+\frac{15 \cos (2 \pi q)}{(2 \pi q)^{6}}-\frac{15 \cos (2 \pi q y)}{(2 \pi q)^{6}}\right) \\
& -y\left(\frac{5}{6(2 \pi q)^{2}}-\frac{5 \sin (2 \pi q)}{2(2 \pi q)^{3}}-\frac{5 \cos (2 \pi q)}{(2 \pi q)^{4}}\right. \\
& \left.+\frac{5 \sin (2 \pi q)}{(2 \pi q)^{5}}-\frac{10 \sin (2 \pi q y)}{(2 \pi q)^{5}}\right) \\
& -y^{2} \frac{5 \cos (2 \pi q y)}{2(2 \pi q)^{4}}-y^{4} \frac{5}{24(2 \pi q)^{2}}
\end{aligned}
$$

and

$$
\begin{aligned}
\varphi_{2}^{l}(y, q)=y( & -\frac{1}{2}+\frac{5}{3(2 \pi q)^{2}}-\frac{5 \sin (2 \pi q)}{(2 \pi q)^{3}} \\
& \left.+-\frac{10 \cos (2 \pi q)}{(2 \pi q)^{4}} \frac{10 \sin (2 \pi q)}{(2 \pi q)^{5}}\right),
\end{aligned}
$$

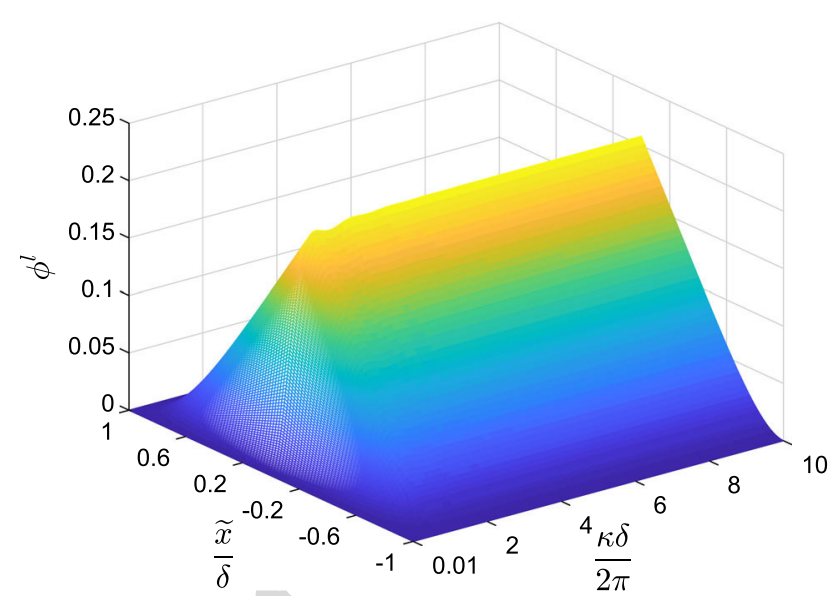

Fig. 7 The function $\Phi^{l}$ on intervals $I_{B} \cup I_{C}$, defined by Eq. (52)

Case of a cubic Morphing function

$$
\begin{gathered}
\alpha(\tilde{x})=0 \quad \forall \tilde{x} \leq 0, \quad \alpha(\tilde{x})=-2(\tilde{x} / L)^{3}+3(\tilde{x} / L)^{2} \forall \tilde{x} \in[0, L], \\
\alpha(\tilde{x})=1 \quad \forall \tilde{x} \geq L .
\end{gathered}
$$

Similar to the above case, Eq.(47) provides the exact analytical form of $\tilde{f}_{g}$ :

$\frac{\tilde{f}_{g}(\tilde{x}, t)}{\rho \ddot{\tilde{u}}(\tilde{x}, t)}=\frac{\delta^{3}}{L^{3}} \Phi_{1}^{c}\left(\frac{\tilde{x}}{\delta}, \frac{\kappa \delta}{2 \pi}\right)+\frac{\delta^{2}}{L^{2}} \Phi_{2}^{c}\left(\frac{\tilde{x}}{\delta}, \frac{\kappa \delta}{2 \pi}\right)$

where

697

698

699

$\Phi_{1}^{c}\left(\frac{\tilde{x}}{\delta}, \frac{\kappa \delta}{2 \pi}\right)=\left\{\begin{array}{lr}0 & \forall \tilde{x} \in I_{A} \\ \varphi_{11}^{c}\left(\frac{\tilde{x}}{\delta}, \frac{\kappa \delta}{2 \pi}\right) & \forall \tilde{x} \in I_{B} \\ \varphi_{11}^{c}\left(\frac{\tilde{x}}{\delta}, \frac{\kappa \delta}{2 \pi}\right)+\varphi_{12}^{c}\left(\frac{\tilde{x}}{\delta}, \frac{\kappa \delta}{2 \pi}\right) & \forall \tilde{x} \in I_{C} \\ -2 \frac{\tilde{x}}{\delta} \psi^{c}\left(\frac{\kappa \delta}{2 \pi}\right) & \forall \tilde{x} \in I_{D}\end{array}\right.$

and

$\Phi_{2}^{c}\left(\frac{\tilde{x}}{\delta}, \frac{\kappa \delta}{2 \pi}\right)= \begin{cases}0 & \forall \tilde{x} \in I_{A} \\ \varphi_{21}^{c}\left(\frac{\tilde{x}}{\delta}, \frac{\kappa \delta}{2 \pi}\right) & \forall \tilde{x} \in I_{B} \\ \varphi_{21}^{c}\left(\frac{\tilde{x}}{\delta}, \frac{\kappa \delta}{2 \pi}\right)+\varphi_{22}^{c}\left(\frac{\tilde{x}}{\delta}, \frac{\kappa \delta}{2 \pi}\right) & \forall \tilde{x} \in I_{C} \\ \psi^{c}\left(\frac{\kappa \delta}{2 \pi}\right) & \forall \tilde{x} \in I_{D}\end{cases}$

with

$$
\begin{aligned}
\varphi_{11}^{c}(y, q)=- & \frac{1}{112}\left(35+120 y+140 y^{2}+56 y^{3}+y^{8}\right) \\
+ & \left(\frac{5}{6(2 \pi q)^{2}}-\frac{5 \sin (2 \pi q)}{(2 \pi q)^{3}}-\frac{25 \cos (2 \pi q)}{(2 \pi q)^{4}}\right. \\
& +\frac{100 \sin (2 \pi q)}{(2 \pi q)^{5}}+\frac{300 \cos (2 \pi q)}{(2 \pi q)^{6}}-\frac{600 \sin (2 \pi q)}{(2 \pi q)^{7}}
\end{aligned}
$$




$$
\begin{aligned}
& \left.-\frac{600 \cos (2 \pi q)}{(2 \pi q)^{8}}+\frac{600 \cos (2 \pi q y)}{(2 \pi q)^{8}}\right) \\
& +y\left(\frac{3}{(2 \pi q)^{2}}-\frac{15 \sin (2 \pi q)}{(2 \pi q)^{3}}-\frac{60 \cos (2 \pi q)}{(2 \pi q)^{4}}\right. \\
& +\frac{180 \sin (2 \pi q)}{(2 \pi q)^{5}}+\frac{360 \cos (2 \pi q)}{(2 \pi q)^{6}} \\
& \left.-\frac{360 \sin (2 \pi q)}{(2 \pi q)^{7}}+\frac{240 \sin (2 \pi q y)}{(2 \pi q)^{7}}\right) \\
& +y^{2}\left(\frac{15}{4(2 \pi q)^{2}}-\frac{15 \sin (2 \pi q)}{(2 \pi q)^{3}}-\frac{45 \cos (2 \pi q)}{(2 \pi q)^{4}}\right. \\
& \left.+\frac{90 \sin (2 \pi q)}{(2 \pi q)^{5}}+\frac{90 \cos (2 \pi q)}{(2 \pi q)^{6}}-\frac{30 \cos (2 \pi q y)}{(2 \pi q)^{6}}\right) \\
& +y^{3}\left(\frac{5}{3(2 \pi q)^{2}}-\frac{5 \sin (2 \pi q)}{(2 \pi q)^{3}}-\frac{10 \cos (2 \pi q)}{(2 \pi q)^{4}}\right. \\
& \left.+\frac{10 \sin (2 \pi q)}{(2 \pi q)^{5}}\right) \\
& +y^{6} \frac{1}{12(2 \pi q)^{2}} \text {, } \\
& \varphi_{12}^{c}(y, q)=y^{3}\left(1-\frac{10}{3(2 \pi q)^{2}}+\frac{10 \sin (2 \pi q)}{(2 \pi q)^{3}}+\frac{20 \cos (2 \pi q)}{(2 \pi q)^{4}}\right. \\
& \left.-\frac{20 \sin (2 \pi q)}{(2 \pi q)^{5}}\right) \text {, } \\
& \varphi_{21}^{c}(y, q)=\frac{1}{28}\left(15+35 y+21 y^{2}+y^{7}\right) \\
& +\left(-\frac{3}{2(2 \pi q)^{2}}+\frac{15 \sin (2 \pi q)}{2(2 \pi q)^{3}}+\frac{30 \cos (2 \pi q)}{(2 \pi q)^{4}}\right. \\
& -\frac{90 \sin (2 \pi q)}{(2 \pi q)^{5}}-\frac{180 \cos (2 \pi q)}{(2 \pi q)^{6}}+ \\
& \left.\frac{180 \sin (2 \pi q)}{(2 \pi q)^{7}}+\frac{180 \sin (2 \pi q y)}{(2 \pi q)^{7}}\right) \\
& +y\left(-\frac{15}{4(2 \pi q)^{2}}+\frac{15 \sin (2 \pi q)}{(2 \pi q)^{3}}+\frac{45 \cos (2 \pi q)}{(2 \pi q)^{4}}\right. \\
& \left.-\frac{90 \sin (2 \pi q)}{(2 \pi q)^{5}}-\frac{90 \cos (2 \pi q)}{(2 \pi q)^{6}}-\frac{90 \cos (2 \pi q y)}{(2 \pi q)^{6}}\right) \\
& +y^{2}\left(-\frac{5}{2(2 \pi q)^{2}}+\frac{15 \sin (2 \pi q)}{2(2 \pi q)^{3}}+\frac{15 \cos (2 \pi q)}{(2 \pi q)^{4}}\right. \\
& \left.-\frac{15 \sin (2 \pi q)}{(2 \pi q)^{5}}-\frac{15 \sin (2 \pi q y)}{(2 \pi q)^{5}}\right) \\
& -y^{5} \frac{1}{4(2 \pi q)^{2}} \text {, } \\
& \varphi_{22}^{c}(y, q)=y^{2}\left(-\frac{3}{2}+\frac{5}{(2 \pi q)^{2}}-\frac{15 \sin (2 \pi q)}{(2 \pi q)^{3}}-\frac{30 \cos (2 \pi q)}{(2 \pi q)^{4}}\right. \\
& \left.+\frac{30 \sin (2 \pi q)}{(2 \pi q)^{5}}\right)
\end{aligned}
$$

730 and

$$
\begin{aligned}
{ }_{731} \psi^{c}(q)= & \frac{15}{14}-\frac{3}{(2 \pi q)^{2}}+\frac{15 \sin (2 \pi q)}{(2 \pi q)^{3}}+\frac{60 \cos (2 \pi q)}{(2 \pi q)^{4}} \\
& -\frac{180 \sin (2 \pi q)}{(2 \pi q)^{5}}-\frac{360 \cos (2 \pi q)}{(2 \pi q)^{6}}+\frac{360 \sin (2 \pi q)}{(2 \pi q)^{7}},
\end{aligned}
$$$$
733
$$

where $y=\tilde{x} / \delta$ and the peridynamic wavenumber $q={ }_{734}$ $\kappa \delta /(2 \pi)$.

Similarly, the functions $\Phi_{1}^{c}$ and $\Phi_{2}^{c}$ in the range $I_{B} \cup I_{C}$ are plotted in Fig. 8 and the function $\psi^{c}$ is plotted in Fig. 9. We can see from these figures that all functions are bounded on their own domains and they are convergent for the large $q$. Additionally, we consider $\tilde{f}_{g}(\tilde{x}, t)$ in the interval $I_{D}$ according to Eq. (56) as follows.

$$
\frac{\tilde{f}_{g}(\tilde{x}, t)}{\rho \ddot{\tilde{u}}(\tilde{x}, t)}=\frac{\delta^{2}}{L^{2}}\left[-\frac{2 \delta}{L} \frac{\tilde{x}}{\delta} \psi^{c}\left(\frac{\kappa \delta}{2 \pi}\right)+\psi^{c}\left(\frac{\kappa \delta}{2 \pi}\right)\right] .
$$

Because $\tilde{x} \in I_{D}=[\delta, L / 2]$, we can conclude that

$0 \leq-\frac{2 \delta}{L} \frac{\tilde{x}}{\delta} \psi^{c}\left(\frac{\kappa \delta}{2 \pi}\right)+\psi^{c}\left(\frac{\kappa \delta}{2 \pi}\right) \leq \psi^{c}\left(\frac{\kappa \delta}{2 \pi}\right)$,

where $\psi^{c}\left(\frac{\kappa \delta}{2 \pi}\right) \geq 0$, referring to Fig. 9. As a result, we can conclude that $\frac{\tilde{f}_{g}(\tilde{x}, t)}{\rho \tilde{u}(\tilde{x}, t)}=O\left(\frac{\delta^{2}}{L^{2}}\right)$ over the whole domain using the cubic Morphing function.

According to the above equations, we can draw the following conclusions:

Compared with the linear Morphing function, The magnitude of the ghost force decreases when the cubic Morphing function is used;

- The magnitude of the ghost force is driven by the ratio, $\delta / L$. When $\delta$ is given but nonzero, the coupling domain must be sufficiently large and its dimensions can be used to control the level of ghost forces;

- The ghost forces begin to vanish when $\delta$ trends towards zero, because a peridynamic model converges towards a classical continuum model in this case [27].

\subsubsection{Work done by ghost forces}

Comparing with the ideal hybrid model, ghost forces are the residuals of the force equilibrium, resulting in the spurious reflections of waves. Therefore, the energy carried by the reflected waves should be equal to the work done by the ghost forces. Since the energy of the entire system is conserved, the work done by ghost forces can be estimated. Thus, we can quantify and bound the drifting energy during wave propagation, and therefore, we can assess the quality of the hybrid local/nonlocal continuum model.

According to Eq. (42), the following integral equation holds using the principle of virtual work:

$$
\begin{aligned}
\int_{\Omega}(\rho \ddot{\boldsymbol{u}}) \cdot \boldsymbol{u} d \Omega= & \int_{\Omega}(\operatorname{div} \boldsymbol{\sigma}) \cdot \boldsymbol{u} d \Omega+\int_{\Omega} \int_{\mathcal{H}_{\delta}} \boldsymbol{f} \cdot \boldsymbol{u} d V d \Omega \\
& +\int_{\Omega} \tilde{\boldsymbol{f}}_{g} \cdot \boldsymbol{u} d \Omega .
\end{aligned}
$$

743$$
735
$$$$
736
$$$$
737
$$$$
738
$$$$
739
$$$$
740
$$$$
41
$$

2

43

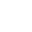

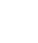

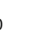

.




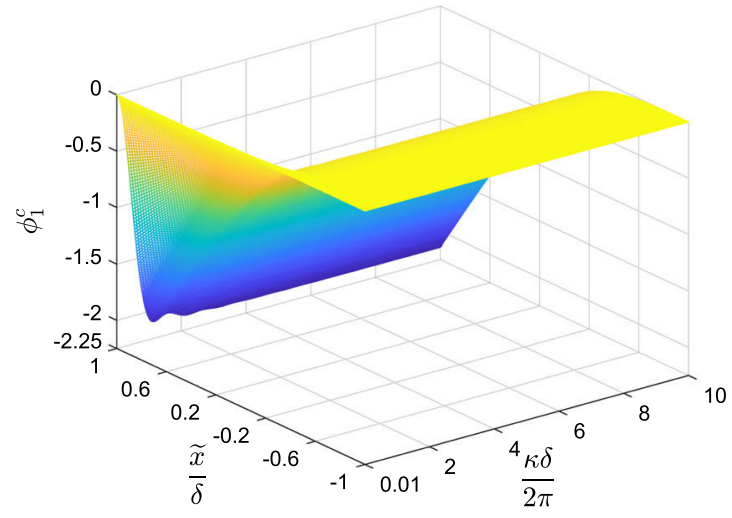

(a)

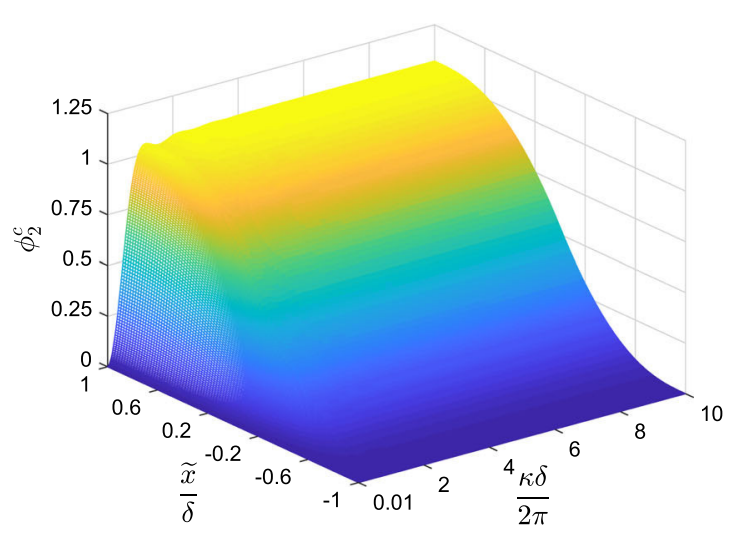

(b)

Fig. 8 The functions $\mathbf{a} \Phi_{1}^{c}$ and $\mathbf{b} \Phi_{2}^{c}$ on intervals $I_{B} \cup I_{C}$, are defined in Eqs. (57) and (58), respectively

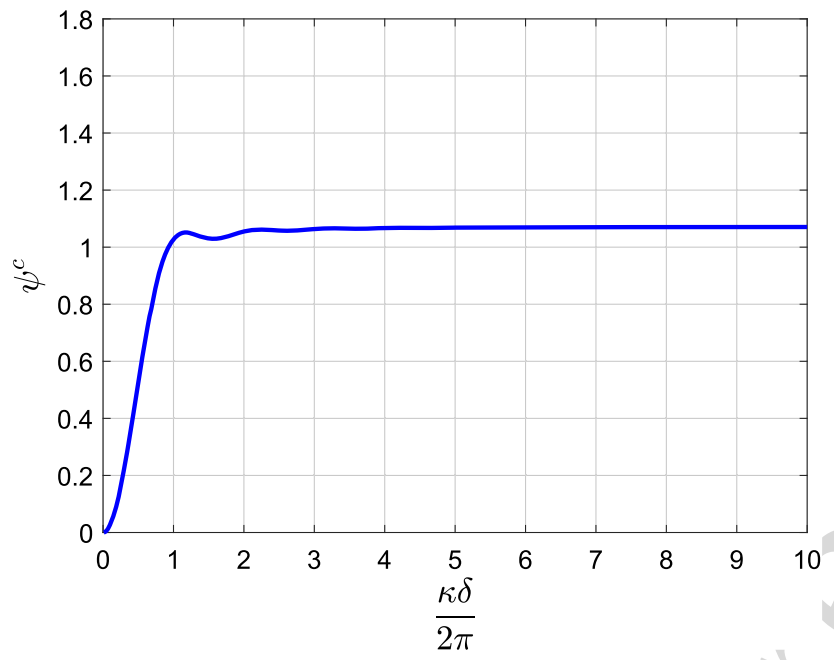

Fig. 9 The function $\psi^{c}$ on interval $[0,10]$, defined in Eq. (63)

Let $\tilde{\mathcal{F}}_{g}=\int_{\Omega} \tilde{\boldsymbol{f}}_{g} \cdot \boldsymbol{u} d \Omega$ be the work done by ghost forces. Similar to the derivation from Eq. (42) to Eq. (45), Eq. (66) can be recast as

$\tilde{\mathcal{F}}_{g}=\int_{\Omega}-\kappa^{2} e^{i 2 \kappa(\boldsymbol{g} \cdot \boldsymbol{x}-v t)}\left(-\alpha(\boldsymbol{x}) \boldsymbol{D}_{c}(\boldsymbol{g})+\alpha(\boldsymbol{x}) \boldsymbol{D}_{p}(\boldsymbol{g}, \kappa)\right.$

$$
\left.+\boldsymbol{D}_{\alpha}(\boldsymbol{g}, \boldsymbol{x}, \kappa)\right) \boldsymbol{a}^{2} d \Omega .
$$

\section{An evaluation in the one-dimensional case}

Considering the 1D case described above, we further evaluate the work done by ghost force. Substituting Eqs. (46) into Eq. (67), it yields as follows:

$\tilde{\mathcal{F}}_{g}=\int_{\Omega} p(x)\left(\rho \dot{\tilde{u}}_{c}(x, t) \dot{\tilde{u}}_{c}(x, t)\right) d \Omega$,

where the function $p(x)$ is defined in Eq. (47) and the following equations are employed according to the $1 \mathrm{D}$ formulation of Eqs. (31) and (22), i.e.,

$$
\begin{aligned}
-E^{0} \kappa^{2} e^{i 2 \kappa(x-v t)} a^{2} & =-\rho \kappa^{2} v_{c}^{2} e^{i 2 \kappa(x-v t)} a^{2} \\
& =\rho \dot{\tilde{u}}_{c}(x, t) \dot{\tilde{u}}_{c}(x, t),
\end{aligned}
$$

where $\tilde{u}_{c}$ denotes the displacement field of a 1D homogeneous bar described by the purely classical continuum model.

Since $\rho \dot{\tilde{u}}_{c}(x, t) \dot{\tilde{u}}_{c}(x, t)>0, \quad \forall x \in \Omega$ and $p(x)$ is a continuous function on $\Omega$, according to the general form of the mean value theorem for integrals, we can ascertain that $\exists x_{0} \in \Omega$, such that

$\tilde{\mathcal{F}}_{g}=p\left(x_{0}\right) \int_{\Omega}\left(\rho \dot{\tilde{u}}_{c}(x, t) \dot{\tilde{u}}_{c}(x, t)\right) d \Omega$.

Since $\tilde{u}_{c}$ denote the displacement field of a 1D homogeneous bar described by the purely classical continuum model, the value of $\int_{\Omega}\left(\rho \dot{\tilde{u}}_{c}(x, t) \dot{\tilde{u}}_{c}(x, t)\right) d \Omega$ is constant. Let $\tilde{\mathcal{K}}=$ $\int_{\Omega}\left(\rho \dot{\tilde{u}}_{c}(x, t) \dot{\tilde{u}}_{c}(x, t)\right) d \Omega$ denote 2 times the kinetic energy of plane wave propagation in the local continuum bar, Eq. (70) can be recast as

$\tilde{\mathcal{F}}_{g}=p\left(x_{0}\right) \tilde{\mathcal{K}}$.

Similar to the above error analysis, we redefine the origin of coordinates $\tilde{x}$ to be the transition point between $\Omega_{m_{1}}$ and $\Omega_{m 0}$, as shown in Fig. 6. The work of ghost forces can then be rewritten as $\tilde{\mathcal{F}}_{g}=p\left(\tilde{x}_{0}\right) \tilde{\mathcal{K}}$. We further discuss the value range of $p\left(\tilde{x}_{0}\right)$ for the hybrid models with different morphing functions:

- For the case with a linear Morphing function discussed above:

$$
p\left(\tilde{x}_{0}\right)=\frac{\delta}{L} \Phi^{l}\left(\frac{\tilde{x}_{0}}{\delta}, \frac{\kappa \delta}{2 \pi}\right)
$$




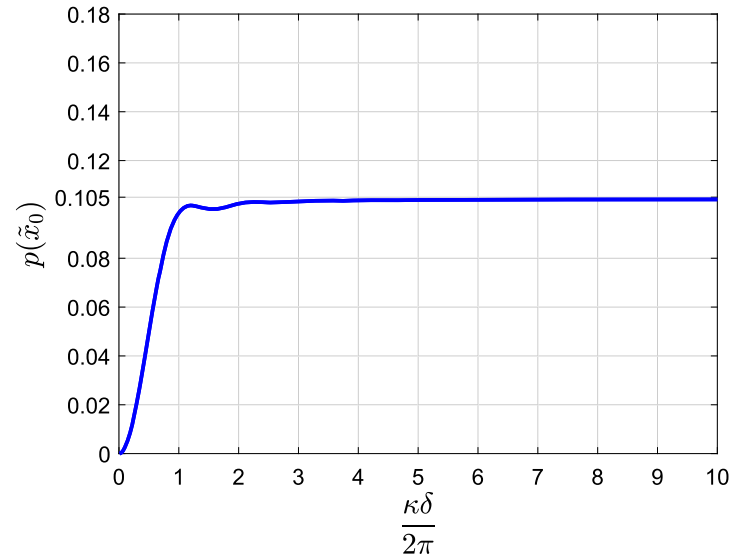

(a) $L=2 \delta$ and $\tilde{x}_{0} / \delta=0$

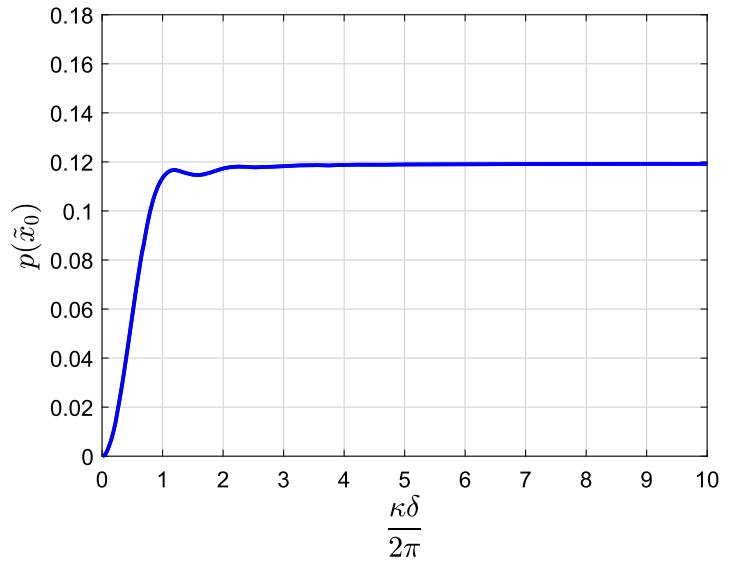

(b) $L=2 \delta$ and $\tilde{x}_{0} / \delta=0.2814$

Fig. 10 a The case with a linear morphing function and $\mathbf{b}$ the case with a cubic Morphing function

where the function $\Phi^{l}$ is defined by Eq. (52). Similar to ghost forces, $\tilde{\mathcal{F}}_{g}=o\left(\frac{\delta}{L}\right)$ due to $\tilde{\mathcal{K}}$ is constant. By numerically analyzing on Eq. (52) and Fig. 7, the peak function of $p\left(\tilde{x}_{0}\right)$ is reached when $L=2 \delta$ and $\tilde{x}_{0} / \delta=0$, which is plotted in Fig. 10a. As can be seen in Fig. 10a, $p\left(\tilde{x}_{0}\right)$ converges to 0.105 as $q=\kappa /(2 \pi / \delta)$ becomes large. Consequently, we can obtain that $0 \leq \tilde{\mathcal{F}}_{g}<0.105 \tilde{\mathcal{K}}$ when $\delta / L=1 / 2$.

- For the case with a cubic Morphing function discussed above:

$p\left(\tilde{x}_{0}\right)=\frac{\delta^{3}}{L^{3}} \Phi_{1}^{c}\left(\frac{\tilde{x}_{0}}{\delta}, \frac{\kappa \delta}{2 \pi}\right)+\frac{\delta^{2}}{L^{2}} \Phi_{2}^{c}\left(\frac{\tilde{x}_{0}}{\delta}, \frac{\kappa \delta}{2 \pi}\right)$,

where the functions $\Phi_{1}^{c}$ and $\Phi_{2}^{c}$ are defined by Eqs. (57) and (58), respectively. Similar to ghost forces, $\tilde{\mathcal{F}}_{g}=$ $o\left(\frac{\delta^{2}}{L^{2}}\right)$ due to $\tilde{\mathcal{K}}$ is constant. By numerically analyzing Eq. (56) and Figs. 8 and 9, the peak function of $p\left(\tilde{x}_{0}\right)$ is reached when $L=2 \delta$ and $\tilde{x}_{0} / \delta=0.2814$, which is plotted in Fig. 10b. As can be seen in Fig. 10b, $p\left(\tilde{x}_{0}\right)$ converges to 0.12 as $q=\kappa /(2 \pi / \delta)$ becomes large. Consequently, we can obtain that $0 \leq \tilde{\mathcal{F}} g<0.12 \tilde{\mathcal{K}}$ when $\delta / L=1 / 2$.

It is worth noting that the maximum of work $\tilde{\mathcal{F}}_{g}$ will be reduced with $\delta / L$ decreasing.

\section{Numerical examples and discussion}

We illustrate the effectiveness of the proposed coupling strategy with some 1D and 2D numerical examples. In Sect. 5.1, we define the ratio of energy drift to the initial energy of an incident wave to indicate the spurious reflections [28]. Then, we use the ratio of energy drift as an indicator to show the effects of peridynamic influence functions [29] and the numerical convergences with grid sizes. In Sect. 5.2, we simulate that an incident wave results in a fracture near a notch in a 2D plate. Additionally, we implemented all examples, including local and nonlocal continuum models, using finite element analysis (see 1). We employ the explicit central difference scheme for time integration of the equation of motion (see 1). We then state the procedure of numerical algorithm in 1. We apply the cubic Morphing function to all examples, and have discussed its one-dimensional analysis of ghost forces in Sect. 4.4.2.

\subsection{D wave propagation}

In this subsection, we analyze the problem of incident wave propagation in a $1 \mathrm{D}$ bar. The geometry and domain partition of this 1D case are shown in Fig. 11, where $L_{t o t}=80 \mathrm{~m}$ denotes the total length of the studied domain, which consists of three types of subdomains: $\Omega_{1} \cup \Omega_{m_{1}}, \Omega_{m_{0}}$ and $\Omega_{2} \cup \Omega_{m_{2}}$, where $\Omega_{m_{0}}=\left\{\boldsymbol{x} \in \Omega_{m}: \mathcal{H}_{\delta}(\boldsymbol{x}) \subset \Omega_{m}\right\}, \Omega_{m_{1}}=\left\{\boldsymbol{x} \in \Omega_{m}\right.$ : $\left.\mathcal{H}_{\delta}(\boldsymbol{x}) \cap \Omega_{1} \neq \emptyset\right\}$ and $\Omega_{m_{2}}=\left\{\boldsymbol{x} \in \Omega_{m}: \mathcal{H}_{\delta}(\boldsymbol{x}) \cap \Omega_{2} \neq \emptyset\right\}$, on which the Morphing function $\alpha=0,0 \leq \alpha \leq 1$ and $\alpha=1$, respectively. Their lengths are $L_{1}=24 \mathrm{~m}, L=6 \mathrm{~m}$ and $L_{2}=20 \mathrm{~m}$ (see Fig. 11). We assume that the bar has a constant cross-sectional area of $0.01 \mathrm{~m}^{2}$. The bar has material parameters given as $E=200 \mathrm{GPa}$ and $\rho=8000 \mathrm{~kg} / \mathrm{m}^{3}$, and the peridynamic horizon is selected as $\delta=0.6 \mathrm{~m}$. The initial displacement field is given by

$u(x)=a_{1} e^{-\left(\frac{x-a_{2}}{a_{3} \pi}\right)^{2}}$, at $t=0 \mathrm{~s}$,

where $a_{1}=0.2 \mathrm{~m}$ and $a_{2}=0 \mathrm{~m}$ for all 1D examples below. Eq. (74) refers to $[28,30]$ with modified parameters. In these $1 \mathrm{D}$ examples, we only study the rightward traveling incident pulse. 


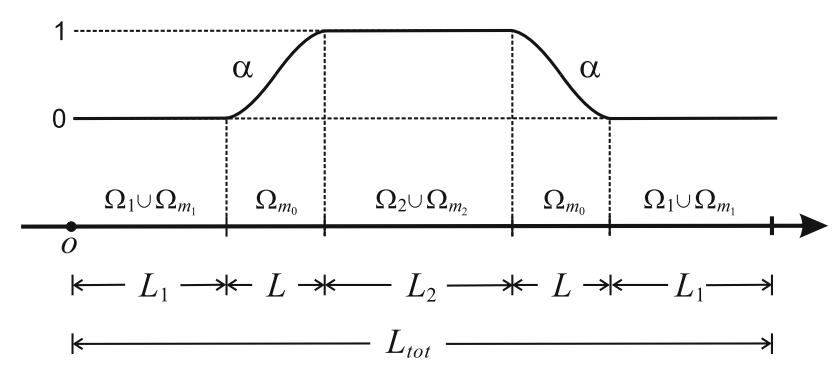

Fig. 11 The subdomain descriptions of the 1D case and the cubic morphing function prescribed on the domain

\subsubsection{The spurious reflection and the ratio of energy drift}

In this example, we study an incident pulse propagating from the left-hand side towards the right-hand side. We assume that the peridynamic micromodulus is $c^{0}(\xi)=\hat{c} e^{-|\xi| / l}$, where $\hat{c}$ is the constant that can be effectively calculated according to the material stiffness $[15,17]$, and $l$ is a characteristic length that is assumed to be $0.12 \mathrm{~m}$. We select the parameter of initial displacement defined in Eq. (74) for this wave to be $a_{3}=1.0 \mathrm{~m}$, which leads to the peridynamic wavenumber of this pulse to be about $q=0.03$. The peridynamic horizon is $0.6 \mathrm{~m}$, as mentioned above, and the pulse width is about $19 \mathrm{~m}$ (inset to Fig. 12a). In this example, the grid size for finite element analysis is $\Delta x=0.1 \mathrm{~m}$, and the time step of $\Delta t=5 \times 10^{-5} \mathrm{~s}$.

Figure 12 shows the snapshots of wave propagation at different times, calculated under the present hybrid local/nonlocal continuum model. As can be seen in Fig. 12a, an incident pulse is located in the purely classical continuum domain (left side) at $t=8 \mathrm{~ms}$. The amplitude and width of the pulse are $0.1 \mathrm{~m}$ and $19 \mathrm{~m}$, respectively. After that, Fig. 12b shows its profile in the other classical continuum domain (right side) at $t=43 \mathrm{~ms}$, which illustrates that this pulse propagates through the coupling domain without significant wave reflections. Zooming in on the bottom of the pulse shows that the spurious reflection is very weak because we only see a few small humps in the peridynamic domain and the absolute values of their crest and trough are less than $1.5 \times 10^{-4} \mathrm{~m}$.

Here, we define a concept of the ratio of energy drift $R_{e d}$ [28] to quantitatively estimate the amount of spurious reflection, which demonstrates the performance of the coupling method. We firstly calculate the energy of pulse $E_{l e f t}$ in the domain $[0,24] \mathrm{m}$ at $t=8 \mathrm{~ms}$ (see Fig. 12a), which includes the elastic deformation energy and kinetic energy of the pulse. Then, we calculate the energy of pulse $E_{\text {right }}$ in the domain $[56,80] \mathrm{m}$ at $t=43 \mathrm{~ms}$ (see Fig. 12b). Finally, the ratio of energy drift is calculated by $R_{e d}=\frac{E_{\text {left }}-E_{\text {right }}}{E_{\text {left }}}$. In this example, the ratio of energy drift is $3.41 \times 10^{-6}$.

\subsubsection{Effect of peridynamic influence function on coupling}

909

In this study, we apply the different peridynamic influence functions to the peridynamic model to analyze the effects of the nonlocal response on the coupling. The computational parameters including grid size, time step, and the initial displacements are the same as those in Sect. 5.1.1. Here, four influence functions are selected: constant, linear and exponential functions with characteristic lengths $l=0.12 \mathrm{~m}$ and $l=0.06 \mathrm{~m}$, respectively denoted by "Exp012" and "Exp006" in Fig. 13a. The shapes of these influence functions are plotted in Fig. 13a. Figure 13b shows the corresponding ratios of energy drift by these four functions. All the ratios are calculated using the same equation and times to those in the above Sect. 5.1.1. As can been seen from Fig. 13b, all the ratios of energy drift are negligible and less than $7 \times 10^{-6}$. The ratio using the constant function is largest due to the strongest nonlocality, and the ratio using the exponential function with the characteristic length of $0.06 \mathrm{~m}$ is smallest due to the relatively reduced horizon which leads to the weakest nonlocality. The other two influence functions, i.e., linear function and exponential function with $l=0.12 \mathrm{~m}$, lead to the ratios of energy drift in the middle ground. These results show that the magnitude of the ratio of energy drift is positively correlated with the strength of nonlocality..

\subsubsection{Convergence analysis on grid size}

In this subsection, the ratio of energy drift is calculated with different grid sizes from $\Delta x=0.2 \mathrm{~m}$ to $\Delta x=0.001 \mathrm{~m}$. Correspondingly, we apply the decreasing time step keeping pace proportionally with the grid size to guarantee numerical stability. For instance, we use the pairs of parameters $\left(\Delta x=0.1 \mathrm{~m}, \Delta t=5 \times 10^{-5} \mathrm{~s}\right)$, $\left(\Delta x=0.008 \mathrm{~m}, \Delta t=4 \times 10^{-6} \mathrm{~s}\right)$ and $(\Delta x=0.001 \mathrm{~m}, \Delta t$ $\left.=5 \times 10^{-7} \mathrm{~s}\right)$ for the simulations. Apart from these parameters, all others, including the influence function, are the same as those used in Sect. 5.1.1. As a result, Fig. 14 shows a clear convergence of the ratio of energy drift with the grid size, and the ratio converges to $3 \times 10^{-6}$.

\subsubsection{The propagation of wave with large wavenumber}

The last example in Sect. 5.1 is the simulation of an incident pulse with a large wavenumber propagating through the hybrid local/nonlocal continuum model. The computational parameters selected here are the same as those used in Sect. 5.1.1, except that $a_{3}$ in the initial displacement Eq. (74) is set to $0.01 \mathrm{~m}$, which results in a very narrow pulse shown in Fig. 15a. The width of this incident pulse at $8 \mathrm{~ms}$ is measured to be only $0.21 \mathrm{~m}$ (see the inset to Fig. 15a). Therefore, the consequent grid size and time step are selected to be $\Delta x=0.001 \mathrm{~m}$ and $\Delta t=5 \times 10^{-7} \mathrm{~s}$ respectively. Since the 


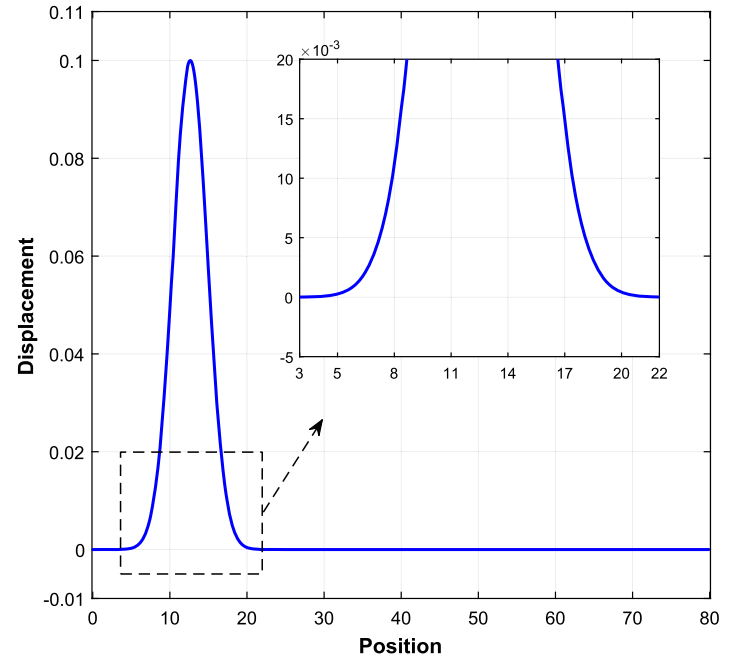

(a) $\mathrm{t}=8 \mathrm{~ms}$

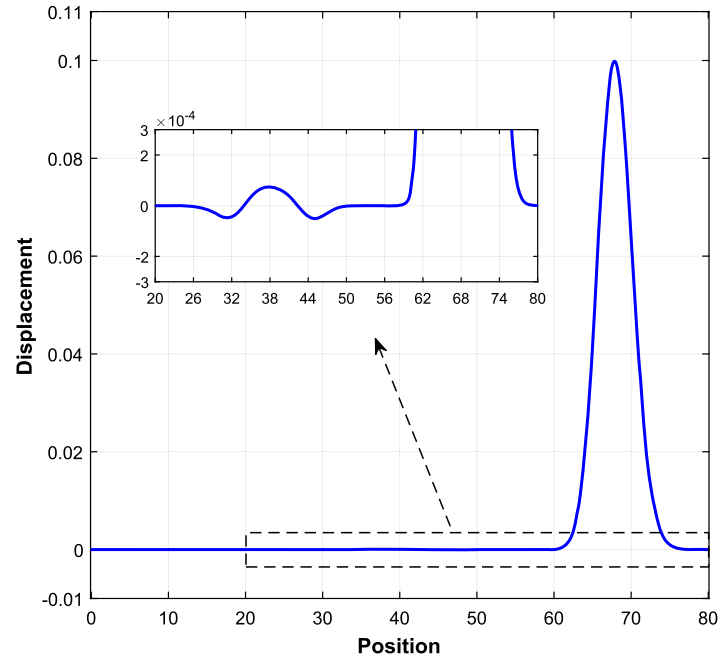

(b) $\mathrm{t}=43 \mathrm{~ms}$

Fig. 12 The snapshots of an incident pulse propagating from left to right at different times, calculated under the hybrid local/nonlocal continuum model (unit of length: $\mathrm{m}$ )

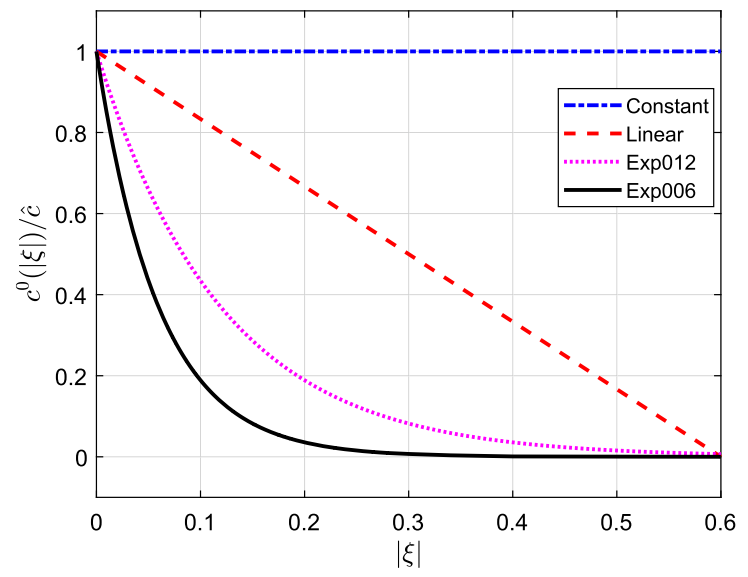

(a)

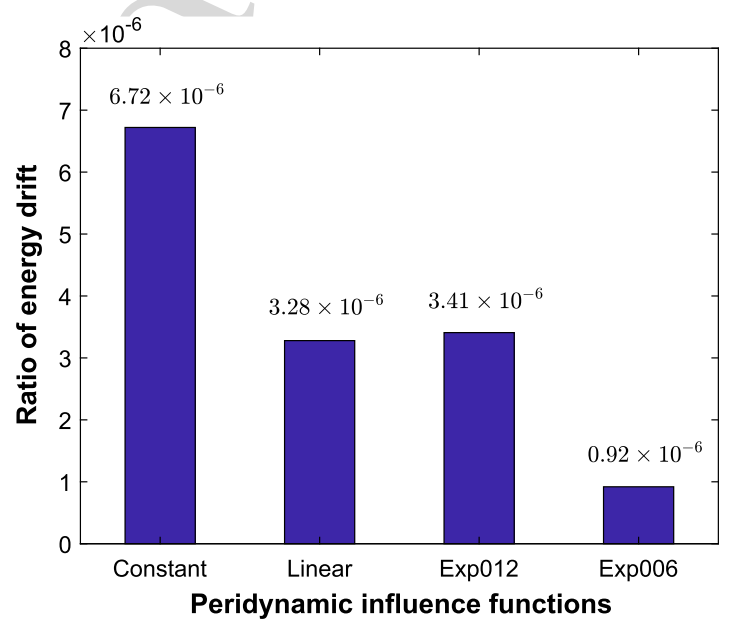

(b)

Fig. 13 a the shapes of peridynamic influence functions and $\mathbf{b}$ the ratio of energy drift for different influence functions

peridynamic horizon is $0.6 \mathrm{~m}$, the peridynamic wavenumber of this pulse is estimated from Fig. 15a to be about $q=2.86$, where $q=\kappa /(2 \pi / \delta)$. With the help of Fig. 4b, we can see that the angular frequency estimated by the peridynamic model is almost equal to the limit value when $q=2.86$. That means the wave with peridynamic wavenumber 2.86 will strongly disperse at the peridynamic domain, as shown in Fig. 15b, c-A at $30 \mathrm{~ms}$. When the incident pulse travels into the purely peridynamics domain, strong dispersion takes place. Nevertheless, the present coupling method still works well. From the zoom-in Fig. 15c-B, we can see the false waves are very small, the absolute values of their crest and trough are less than $3 \times 10^{-6} \mathrm{~m}$, compared with the peak of the initial pulse in Fig. 15a, which is $0.1 \mathrm{~m}$. Unfortunately, it is impossible to accurately estimate the ratio of energy drift, due to the strong dispersions and extremely narrow widths of waves.

\subsection{D wave propagation on a notched plate}

In this section, we numerically study the wave propagation on a $2 \mathrm{D}$ elastic plate with an inner notch. The right end of the rectangular 2D elastic plate is shown in Fig. 16 and the left side is set to extend infinitely. The thickness of the plate is assumed to be $0.01 \mathrm{~m}$. As shown in Fig. 16, the width of plate is $P_{W}=40 \mathrm{~m}, o$ denotes the origin and the length from $o$ to the right edge is $P_{L}=80 \mathrm{~m}$. Both the circular area $\Omega_{2} \cup \Omega_{m_{2}}$ and the circle ring $\Omega_{m_{0}}$ are in the center of the display area, i.e., $(x, y)=(40,20)$. In addition, there exists a narrow notch at the center of the circular area, where the radii are $l_{a}=10 \mathrm{~m}$ 


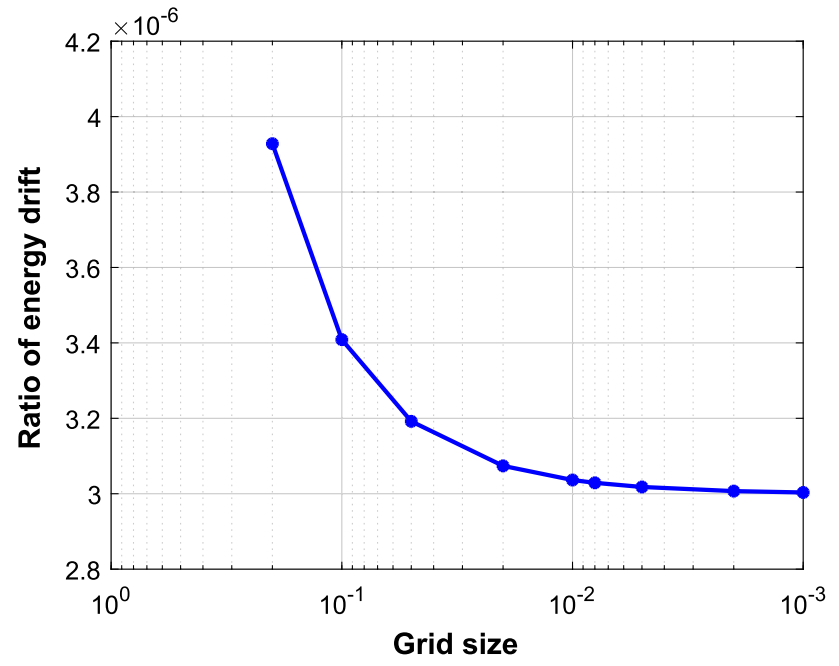

Fig. 14 The ratio of energy drift versus the grid size (unit of length: $\mathrm{m}$ )

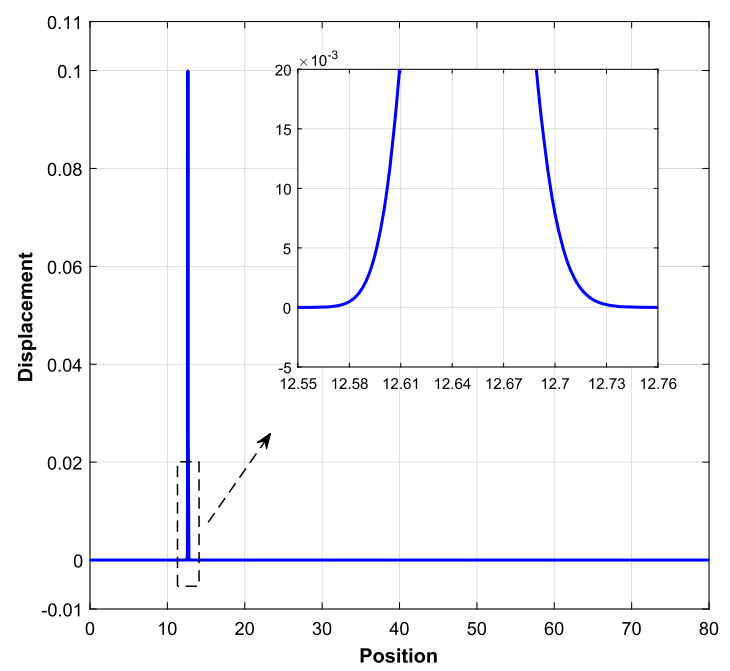

(a) $\mathrm{t}=8 \mathrm{~ms}$ and $l_{b}=13.6 \mathrm{~m}$. The width and length of the notch are $n_{w}=$ $0.4 \mathrm{~m}$ and $n_{l}=6 \mathrm{~m}$, respectively. The angle of notch with the horizontal direction is denoted by $\theta$. Two cases including $\theta=$ 0 (i.e., horizontal notch) and $\theta=\pi / 2$ (i.e., vertical notch), are studied in this paper. The boundary conditions are also shown in Fig. 16; the displacements along vertical direction are constrained for both the horizontal edges while on the right vertical edge the displacements along the horizontal direction are fixed [31].

Here a plane stress assumption is considered, and the plate has an anisotropic material stiffness of Young's moduli $E_{x}=200 \mathrm{GPa}$ and $E_{y}=100 \mathrm{GPa}$ and Poisson's ratio $v=1 / 3$. The material density is $\rho=8000 \mathrm{~kg} / \mathrm{m}^{3}$. Let's consider an exponential function that is the same as that in Sect. 5.1.1 for micromodulus expression. In addition, for an anisotropic material, the micromodulus coefficient function $c^{0}(\xi)$ is also a function of the angles between the bond $\boldsymbol{\xi}$ and the coordinate axes, refer to [17] for a more detailed dis-

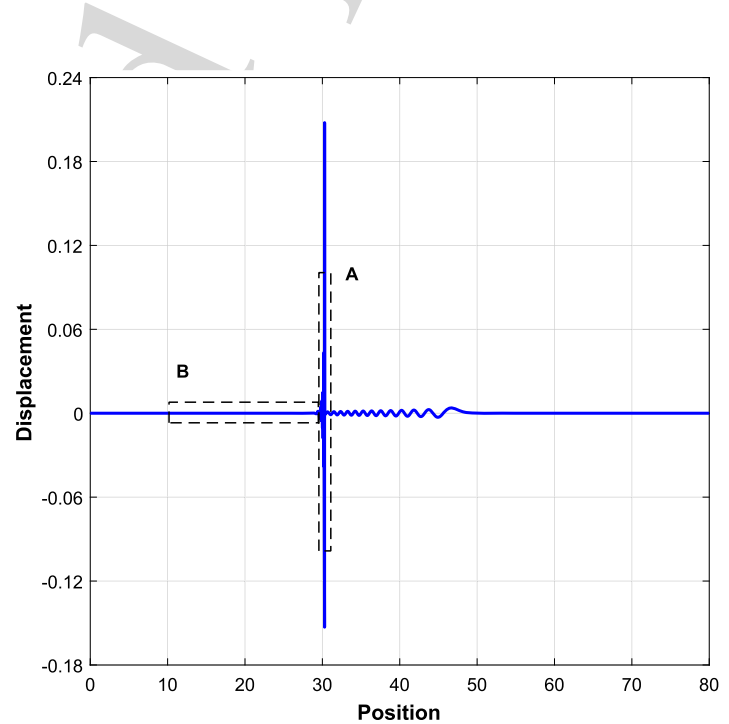

(b) $\mathrm{t}=30 \mathrm{~ms}$
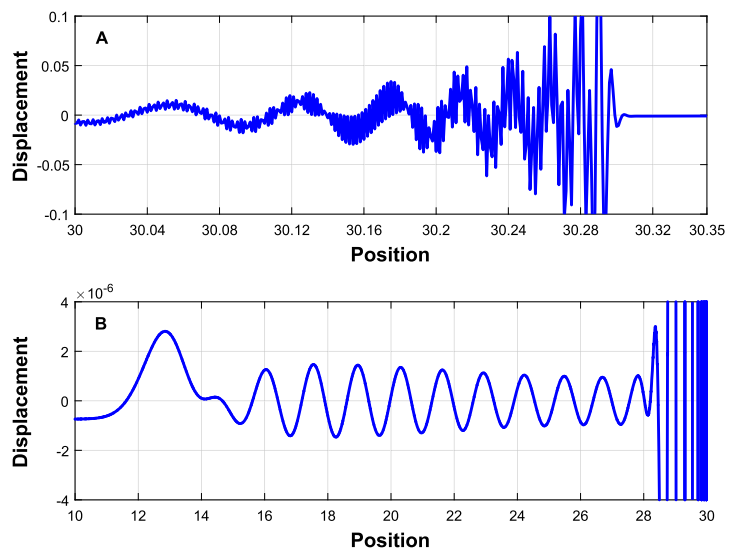

(c) A. local dispersion, B. false waves

Fig. 15 The snapshots of a propagating pulse with large wavenumber, calculated under the hybrid local/nonlocal continuum model (unit of length: m) 
Fig. 16 The geometry and subdomain descriptions of a 2D plate for dynamic simulations (unit of length: $\mathrm{m}$ )

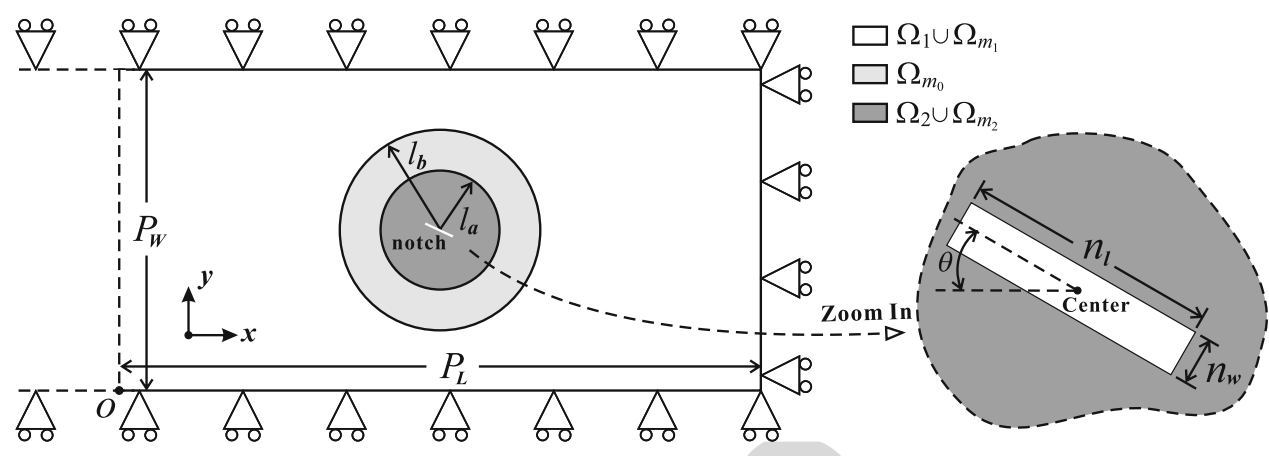

cussion. The characteristic length $l$ is assumed to be $0.12 \mathrm{~m}$ and the peridynamic horizon is $\delta=0.6 \mathrm{~m}$. The plate is discretized by bilinear quadrilateral finite elements (BQFEs) with the size of $0.2 \mathrm{~m}$. Those BQFEs for discretizing the purely peridynamic domain, i.e., $\Omega_{2} \cup \Omega_{m_{2}}$ (see Fig. 16) are set as the discontinuous Galerkin finite elements (DGFEs) $[18,19]$ in all simulations below. Beyond that, the initial displacement field is defined by

$\left\{\begin{array}{l}u_{x}(x, y)=a_{1} e^{-\left(\frac{x-a_{2}}{a_{3} \pi}\right)^{2}} \\ u_{y}(x, y)=0\end{array}\right.$, at $t=0 \mathrm{~s}$,

where $a_{1}=0.1 \mathrm{~m}, a_{2}=0 \mathrm{~m}$, and $a_{3}=1.0 \mathrm{~m}$. The time step of $\Delta t=1 \times 10^{-4} \mathrm{~s}$. Similar to the examples in Sect. 5.1, we only study the rightward traveling incident wave.

Figure 17 shows the displacement fields, $u_{x}$, of the wave propagation at the time $t=45 \mathrm{~ms}$ on the plate with a vertical notch (see Fig. 17a, b) or with a horizontal notch (see Fig. 17c, d), which are calculated by the hybrid local/nonlocal continuum model (see Fig. 17a, c) and by the purely classical continuum model (see Fig. 17b, d). For the sake of comparison, we do not consider bond failures in the current pecirdynamic simulations. As can be seen in Fig. 17, the simulation results of the hybrid model agree with those of the purely classical continuum model, both when the plate has a vertical or horizontal notch. This illustrates that the hybrid model proposed in this paper can also correctly solve the wave propagation problem for $2 \mathrm{D}$ anisotropic plates, even with an inner notch.

However, when the bond breaks are considered by using a bond failure criterion, this hybrid local/nonlocal continuum model can further simulate the process of nucleation and propagation of cracks around a notch caused by wave propagations. These simulation results are shown as follows, in which the critical stretch of a bond [3] is set to $2 \%$. Figure 18 shows the displacement fields, $u_{x}$, of wave propagation and the damage contours near the notch, respectively, at the time $t=45 \mathrm{~ms}$ on the plate with a vertical notch (see Fig. 18a, b) or with a horizontal notch (see Fig. 18c, d). Compared with Fig. 17a, c, respectively, Fig. 18a, c shows the same displacement fields at the right end of the plate, which represents the wave pattern of the incident pulse at the time $t=45 \mathrm{~ms}$, but at the left part of the plate, especially near the notch, the displacement fields are distinctly different. This is because the wave propagation causes some bonds near the notch to break, which results in the local damage of the material, which further affects the simulation results of the displacement field. The zoomed-in damage contours at the time $t=45 \mathrm{~ms}$ near the notches are shown in Fig. 18b, d, respectively. By comparing Fig. 18a, c, we can see that when a bar-shaped notch is perpendicular to the propagation direction of the plane wave, the long side of the notch as a free boundary reflects more incident waves. In contrast, when the bar-shaped notch is parallel to the propagation direction of the plane wave, the short side of the notch reflects fewer incident waves, resulting in little change in the plane wave after passing through the notch. Moreover, as shown in Figs. 18b, d, when the barshaped notch is perpendicular to the propagation direction of the plane wave, a severe and large area of damage appears before the notch. However, when the bar-shaped notch is parallel to the direction of wave propagation, minor damage is caused near both ends of the notch only.

We are interested in how this damage occurs during the propagation of waves. We first study case when the plane wave is passing through the vertical notch. Figure 19a, b shows the snapshots of displacement field $u_{x}$ and damage contour, respectively, at the time $t=26 \mathrm{~ms}$ around the vertical notch. Figure 19b shows that two long parallel cracks appear in the damaged area before the bar-shaped notch. This type of long parallel crack is considered to be a common pattern of dynamic tensile damage (spallation) caused by shock loading $[12,32,33]$. From Fig. 19a, indicates that mode of failure is the reflection of the compression shock wave (i.e., the incident wave) from the left free edge of the notch that results in a rarefaction wave, which interacts with the tail part of the incident shock wave (also a rarefaction wave) to form tensile load, which then leads to the formation of cracks.

Then, we study another case when the plane wave is passing through the horizontal notch. Figure 20 shows the snapshots of displacement fields $u_{x}$ and damage contours around the horizontal notch at the times $t=26 \mathrm{~ms}$ and $t=29.5 \mathrm{~ms}$, respectively. Compared with Fig. 19, the dam- 


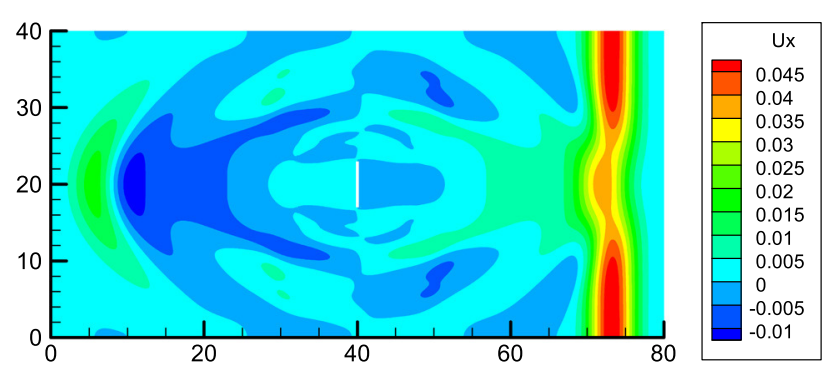

(a) $u_{x}$ on the plate with a vertical notch
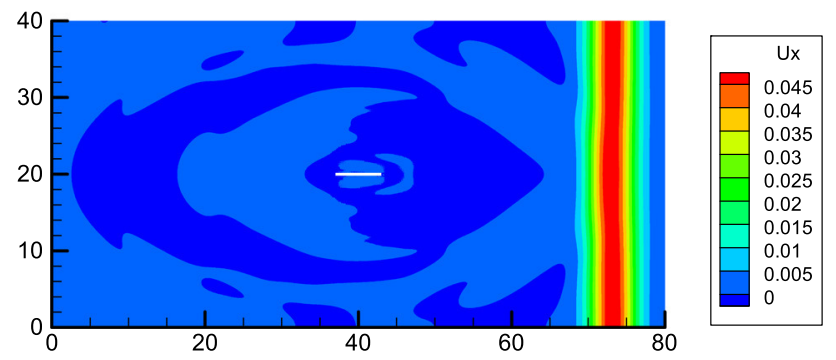

(c) $u_{x}$ on the plate with a horizontal notch

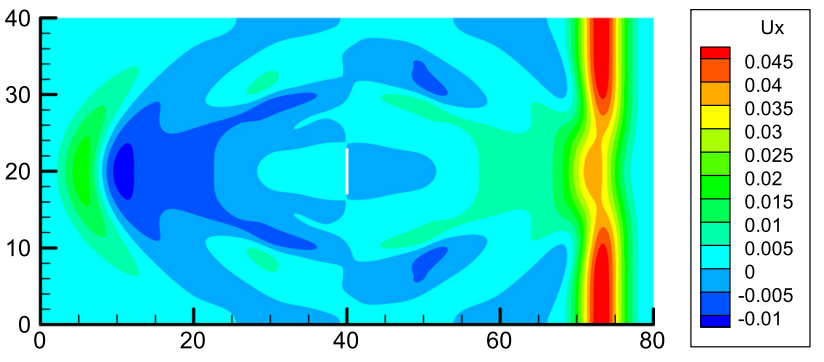

(b) $u_{x}$ on the plate with a vertical notch

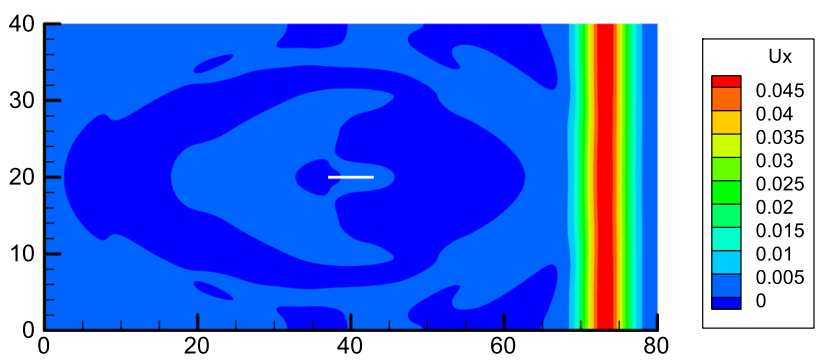

(d) $u_{x}$ on the plate with a horizontal notch

Fig. 17 The displacement fields of wave propagation at $45 \mathrm{~ms}$ for the different models: a, $\mathbf{c}$ the hybrid local/nonlocal continuum model without using bond failure criterion, $\mathbf{b}, \mathbf{c}$ the purely classical continuum model (unit of length and displacement: $\mathrm{m}$ )
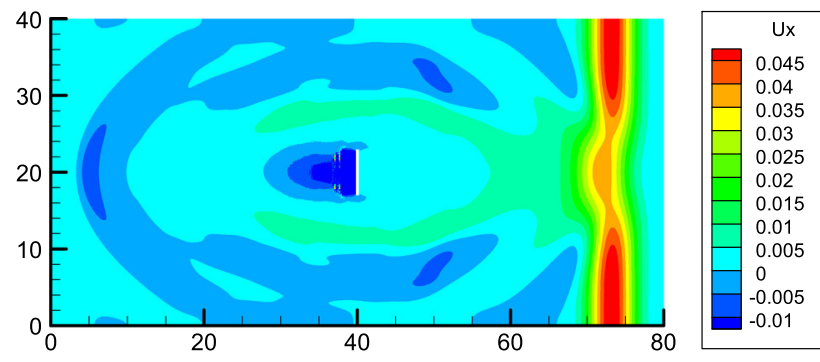

(a) $u_{x}$ on the plate with a vertical notch
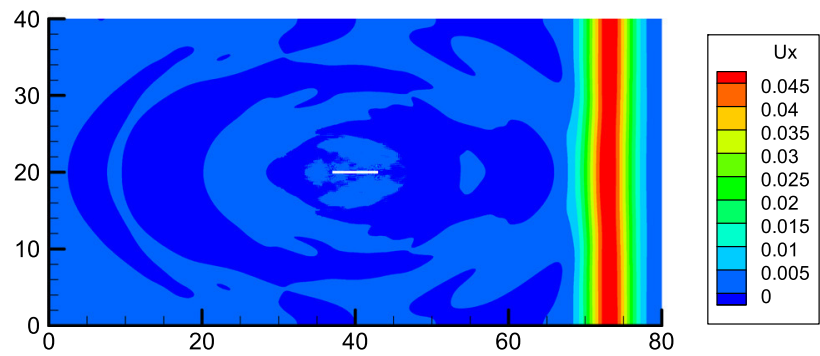

(c) $u_{x}$ on the plate with a horizontal notch
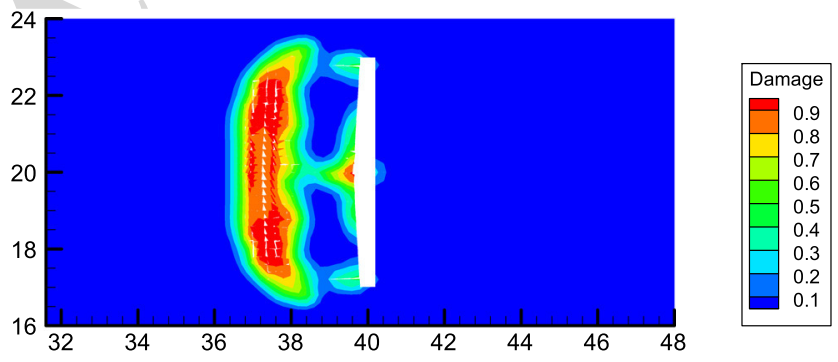

(b) damage contour around vertical notch
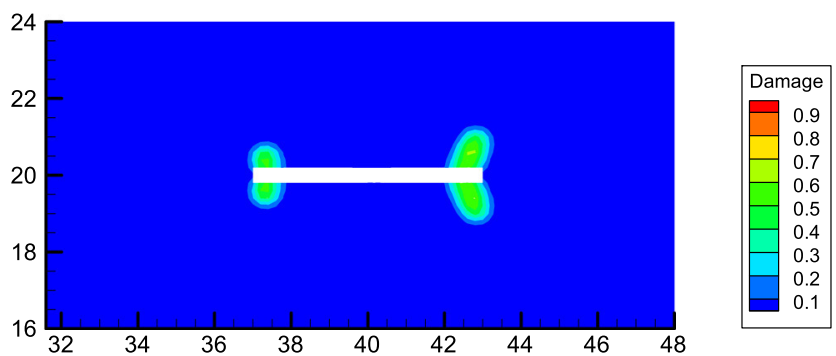

(d) damage contour around horizontal notch

Fig. 18 The snapshots of wave propagation at $45 \mathrm{~ms}$ for the hybrid local/nonlocal continuum model using the bond failure criterion: a, c are the displacement fields, $\mathbf{b}, \mathbf{d}$ are the zoomed-in damage contours around notch (unit of length and displacement: $\mathrm{m}$ ) 


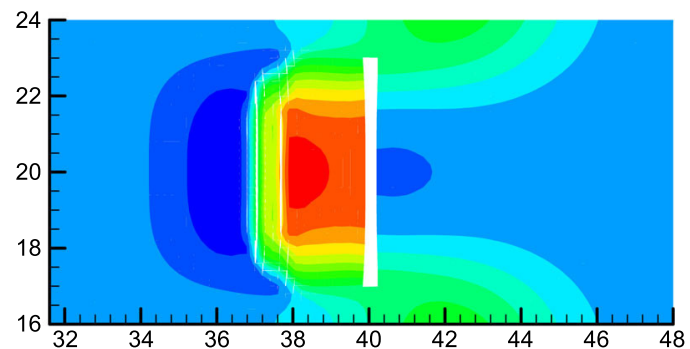

(a) $u_{x}$ at $26 \mathrm{~ms}$
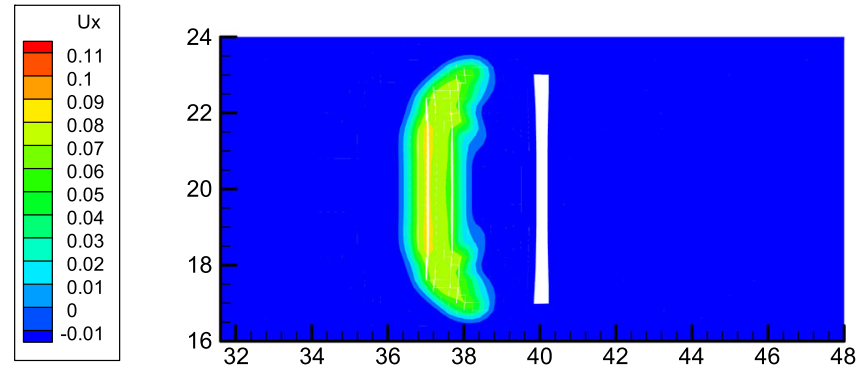

(b) damage contour at $26 \mathrm{~ms}$

Fig. 19 The snapshots of wave propagation at $26 \mathrm{~ms}$ around vertical notch for the hybrid local/nonlocal continuum model using a bond failure criterion: a the zoomed-in displacement fields, $\mathbf{b}$ the zoomed-in damage contours (unit of length and displacement: $\mathrm{m}$ )

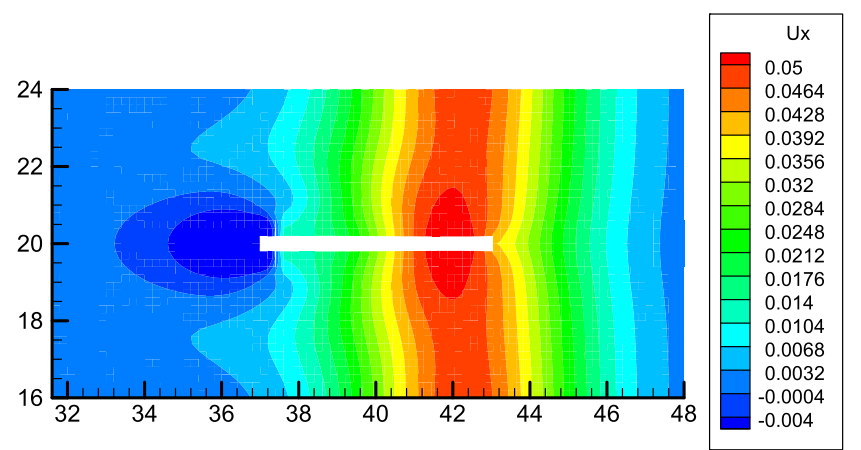

(a) $u_{x}$ at $26 \mathrm{~ms}$

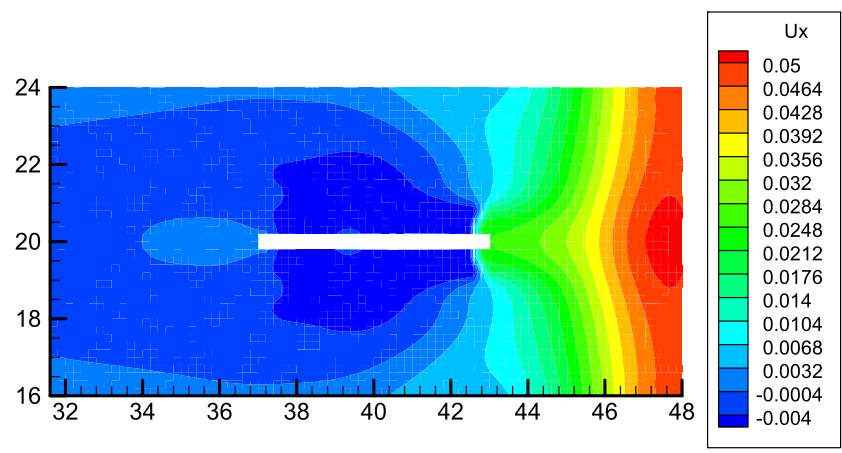

(c) $u_{x}$ at $29.5 \mathrm{~ms}$

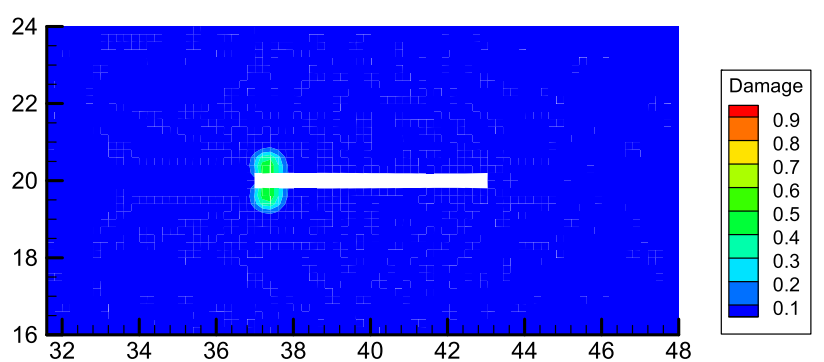

(b) damage contour at $26 \mathrm{~ms}$
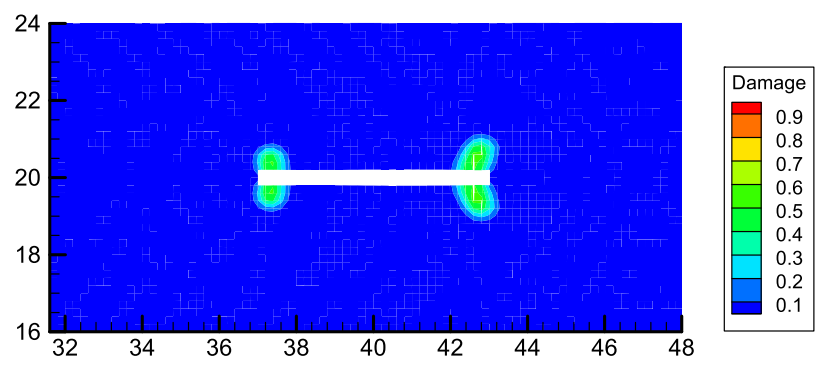

(d) damage contour at $29.5 \mathrm{~ms}$

Fig. 20 The snapshots of wave propagation around a horizontal notch for the hybrid local/nonlocal continuum model using a bond failure criterion: $\mathbf{a}, \mathbf{c}$ are the zoomed-in displacement fields, $\mathbf{b}, \mathbf{d}$ are the zoomed-in damage contours (unit of length and displacement: $\mathrm{m}$ )

age mode in Fig. 20b, d is quite different from that in Fig. 19b. In Fig. 20, the two long free edges of the notch is parallel to the direction of incident wave propagation. The damage is caused by reflected rarefaction waves only near the corners of the notch (i.e., the ends of the notch). Besides, the gradients of the displacement field near the notch are smaller than those in Fig. 19a. The resulting tensile load is much smaller. Thus, the damage is minor and its regions are smaller.

\section{Conclusion}

1089

Here, we developed a dynamic hybrid local/nonlocal continuum model based on the Morphing coupling method. We used this hybrid model to analyze and simulate wave propagation that may result in dispersions or cracks. The theoretical analysis for a plane harmonic wave derives the coupling errors caused by ghost forces, which helps to understand the effects of coupling parameters on the spurious reflection. The energy of the reflected wave is considered equal to the work of the ghost force. The ghost force and its work are 
driven in the $1 \mathrm{D}$ case by the ratio of the peridynamic horizon to the size of the overlapping domain. The ghost force decreases while using a higher order of Morphing function. The work done by the ghost force is much smaller than the kinetic energy of the incident plane wave in the local continuum model. The numerical results demonstrate that the ratio of energy drift, which represents the intensity of spurious reflection, is negligible and convergent with the grid size. The wave dispersions become violent when waves with large wavenumber (i.e., high-frequency wave) travel in the peridynamic domain. At that time, however, the spurious reflection is still small. When a wave propagates near a notch in the 2D plate, it may cause dynamic tensile damages like spallation, if bond breakage is considered in the simulation.

Acknowledgements The authors gratefully acknowledge the financial support received from the National Natural Science Foundation (11872016), the Fundamental Research Funds of Dalian University of Technology (Grant No. DUT20RC(5)005), and KAUST baseline funding for the completion of this work. Helpful discussions with Dr. Youwei Zhang from Dalian University of Technology and with Dr. Meizhen Xiang from Laboratory of Computational Physics, Institute of Applied Physics and Computational Mathematics are also gratefully acknowledged.

\section{Appendix A: Numerical discretization and algorithm procedure}

The numerical discretization and algorithm procedure for the hybrid equations of motion (i.e., Eqs. (6)-(13)) is stated below. We consider the spatial and temporal variables separately. The equations are first discretized on the spatial dimension by the finite element method. The approach is the same as for the static analysis. Then, the resulting equation, which involves the second derivative of displacement with respect to time, is discretized on the temporal dimension by the finite difference method. Finally, we list the algorithm procedures to solve the hybrid equation of motion.

\section{Appendix A.1: The spatial discretization by finite element method}

We divide the whole domain, $\Omega$, by a finite number of elements, $V_{i}, i=1,2, \ldots, n$, where $n$ is the number of elements. These elements are nonoverlapping, but common vertices, called "mesh nodes", are shared between adjacent elements. Thus, we can write that $\Omega=V_{1} \cup V_{2} \cup \cdots \cup V_{n}$. Because it is defined in Sect. 3 that $\Omega=\Omega_{1} \cup \Omega_{m} \cup \Omega_{2}$, $\Omega_{1} \cap \Omega_{2}=\emptyset, \Omega_{1} \cap \Omega_{m}=\emptyset$ and $\Omega_{2} \cap \Omega_{m}=\emptyset$, we assume for definiteness and without a loss of generality that $\Omega_{1}=$ $V_{1} \cup V_{2} \cup \cdots \cup V_{n^{\prime}}, \Omega_{m}=V_{n^{\prime}+1} \cup V_{n^{\prime}+2} \cup \cdots \cup V_{n^{\prime \prime}}$ and $\Omega_{2}=$ $V_{n^{\prime \prime}+1} \cup V_{n^{\prime \prime}+2} \cup \cdots \cup V_{n}$, for $1<n^{\prime}<n^{\prime \prime}<n$. In addition, we define the divisions of the neighborhood of any point $x$ (i.e.,
$\left.\mathcal{H}_{\delta}(\boldsymbol{x}) \cap \Omega\right)$. Indeed, there exists a minimal set of elements, $\mathcal{A}_{x}$, which is defined by $\mathcal{A}_{x}=\left\{V_{x}^{1}, V_{x}^{2}, \ldots, V_{x}^{h(x)}\right\} \subset$ $\left\{V_{1}, V_{2}, \ldots, V_{n}\right\}$, such that $\left(\mathcal{H}_{\delta}(\boldsymbol{x}) \cap \Omega\right) \subset \mathcal{B}_{x}, \forall \boldsymbol{x} \in \Omega \backslash \Omega_{1}$, where $\mathcal{B}_{x}=V_{x}^{1} \cup V_{x}^{2} \cdots \cup V_{x}^{h(x)}$ and $h(x)$ denotes the number of elements in $\mathcal{A}_{\boldsymbol{x}}$. By reassigning a value of 0 to $c^{0}(|\boldsymbol{\xi}|)$, where $\boldsymbol{\xi}=\boldsymbol{x}^{\prime}-\boldsymbol{x}$, for $\boldsymbol{x}^{\prime} \in \mathcal{B}_{\boldsymbol{x}} \backslash \mathcal{H}_{\delta}(\boldsymbol{x})$, the coefficient function of micromodulus $c^{0}(|\xi|)$ is extended to the whole domain $\mathcal{B}_{x}$. Then, a virtual work equation corresponding to Eqs. (6)(13) can be written by replacing the subdomain $\left(\mathcal{H}_{\delta}(\boldsymbol{x}) \cap \Omega\right)$ with the subdomain $\mathcal{B}_{x}$ for integration. That is

$$
\begin{aligned}
& \int_{\Omega} P(\boldsymbol{u}, t) d \Omega+\int_{\Omega} W_{c}(\boldsymbol{\varepsilon}, t) d \Omega+\int_{\Omega} W_{p}(\boldsymbol{\eta}, t) d \Omega \\
& =\int_{\Omega} Q_{b}(\boldsymbol{u}, t) d \Omega+\int_{\Gamma_{T}} Q_{T}(\boldsymbol{u}, t) d S
\end{aligned}
$$

where

$P(\boldsymbol{u}, t)=\boldsymbol{u}(\boldsymbol{x}, t) \cdot(\rho(\boldsymbol{x}) \ddot{\boldsymbol{u}}(\boldsymbol{x}, t))$,

$W_{c}(\boldsymbol{\varepsilon}, t)=\frac{1}{2} \boldsymbol{\varepsilon}(\boldsymbol{x}, t): \boldsymbol{E}(\boldsymbol{x}): \boldsymbol{\varepsilon}(\boldsymbol{x}, t)$,

$W_{p}(\boldsymbol{\eta}, t)=\frac{1}{4} \int_{\mathcal{B}_{x}} c^{0}(|\boldsymbol{\xi}|) \frac{\alpha(\boldsymbol{x})+\alpha\left(\boldsymbol{x}^{\prime}\right)}{2}\left(\boldsymbol{\xi} \cdot \boldsymbol{\eta}\left(\boldsymbol{x}^{\prime}, \boldsymbol{x}, t\right)\right)^{2} d V_{\boldsymbol{x}^{\prime}}$

$Q_{b}(\boldsymbol{u}, t)=\boldsymbol{u}(\boldsymbol{x}, t) \cdot \boldsymbol{b}(\boldsymbol{x}, t)$, and

$Q_{T}(\boldsymbol{u}, t)=\boldsymbol{u}(\boldsymbol{x}, t) \cdot \overline{\boldsymbol{T}}(\boldsymbol{x}, t)$.

The displacement solution can be approximately expressed in the finite element scheme using piecewise interpolation techniques. Let $\boldsymbol{u}_{i}$ denote the displacement solution over the element $V_{i}$, which is given by

$\boldsymbol{u}_{i}(\boldsymbol{x}, t)=\boldsymbol{N}_{i}(\boldsymbol{x}) \boldsymbol{d}_{i}(t), \quad \forall i=1,2, \ldots, n$

where $N_{i}$ is the matrix of shape functions and $\boldsymbol{d}_{i}$ is the nodal displacement. Furthermore, let the number of mesh nodes in the global discretized domain be $m$, then all nodal displacements can be denoted as $\boldsymbol{d}^{\mathrm{T}}=\left\{d_{1}, d_{2}, \ldots, d_{m}\right\}$. Thus, for the nodal displacement $\boldsymbol{d}_{i}$ in any element $V_{i}$, we know that $\boldsymbol{d}_{i} \subset \boldsymbol{d}, \quad \forall i=1,2, \ldots, n$. Moreover, one can define that

$\boldsymbol{d}_{i}(t)=\boldsymbol{R}_{i} \boldsymbol{d}(t), \quad \forall i=1,2, \ldots, n$,

where $\boldsymbol{R}_{i}$ is a diagonal matrix in which the diagonal entries may be 0 or 1 , depending on the nodes of element $V_{i}$. Substituting Eq. (A.3) into the Eq. (A.2), it yields

$\boldsymbol{u}_{i}(\boldsymbol{x}, t)=\boldsymbol{N}_{i}(\boldsymbol{x}) \boldsymbol{R}_{i} \boldsymbol{d}(t), \quad \forall i=1,2, \ldots, n$.

Then, we use the variational method to Eq. (A.1) and, by substituting Eq. (A.4), it can be recast as follows:

$\boldsymbol{M} \ddot{\boldsymbol{d}}(t)+\boldsymbol{K} \boldsymbol{d}(t)=\boldsymbol{F}(t)$, 
$\dot{\boldsymbol{d}}(t)=\frac{1}{2 \Delta t}[-\boldsymbol{d}(t-\Delta t)+\boldsymbol{d}(t+\Delta t)]$.

where $\boldsymbol{H}$ denotes a matrix of differential operators (see $\mathrm{Eq}$ (25)), the notations $[\cdot]$ and $\{\cdot\}$ denote a matrix and a vector, respectively, and $S_{i}$ is the boundary of $V_{i}$.

\section{Appendix A.2: The temporal discretization by finite difference method}

Here, the central difference method is employed. The nodal acceleration $\ddot{\boldsymbol{d}}(t)$ can be approximated by the displacements, that is

$\ddot{\boldsymbol{d}}(t)=\frac{1}{\Delta t^{2}}[\boldsymbol{d}(t-\Delta t)-2 \boldsymbol{d}(t)+\boldsymbol{d}(t+\Delta t)]$,

where $\Delta t$ denotes the time step. Substituting the approximation of the acceleration, (i.e., Eq. (A.6)) into Eq. (A.5) yields

$$
\frac{\boldsymbol{M}}{\Delta t^{2}} \boldsymbol{d}(t+\Delta t)=\boldsymbol{F}(t)-\left(\boldsymbol{K}-\frac{2 \boldsymbol{M}}{\Delta t^{2}}\right) \boldsymbol{d}(t)-\frac{\boldsymbol{M}}{\Delta t^{2}} \boldsymbol{d}(t-\Delta t) .
$$

According to the above Yes, we have explained that it is an approximation, but in order to facilitate the derivation subsequently, we still use the expression of equality., the displacement solution at time $t+\Delta t$ can be solved once the displacements at the last two steps, i.e., $\boldsymbol{d}(t)$ and $\boldsymbol{d}(t-\Delta t)$, have been obtained.

Note that we need to consider the initial conditions for Eq. (A.7). That is, when $t=0$, we need both an initial displacement $\boldsymbol{d}(0)$ and a value of $\boldsymbol{d}(-\Delta t)$ in order to calculate the next step displacement $\boldsymbol{d}(\Delta t)$. According to the central difference approximation, a nodal velocity can be expressed as follows:
Considering both Eqs. (A.6) and (A.8), when $t=0$, the following equation holds:

$\boldsymbol{d}(-\Delta t)=\boldsymbol{d}(0)-\Delta t \dot{\boldsymbol{d}}(0)+\frac{\Delta t^{2}}{2} \ddot{\boldsymbol{d}}(0)$,

where $\ddot{\boldsymbol{d}}(0)$ can be calculated via Eq. (A.5) when $t=0$, i.e., $\quad{ }_{1222}$ $\ddot{\boldsymbol{d}}(0)=\boldsymbol{M}^{-1}[\boldsymbol{F}(0)-\boldsymbol{K} \boldsymbol{d}(0)]$.

Consequently, the initial velocity $\dot{\boldsymbol{d}}(0)$ is necessary to replace $\quad 1224$ the value $\boldsymbol{d}(-\Delta t)$ when $t=0$. Thus, both displacement $\boldsymbol{d}(0)$ and velocity $\dot{\boldsymbol{d}}(0)$ are the initial conditions for Eq. (A.7). $\quad{ }_{1226}$

\section{Appendix A.3: Numerical algorithm procedure}

We rewrite Eq. (A.7) into a form of linear equation as follows:

1. Assemble mass matrix $\boldsymbol{M}$ and stiffness matrix $\boldsymbol{K}$.

where $\hat{\boldsymbol{M}}=\boldsymbol{M} / \Delta t^{2}, \boldsymbol{d}_{t+\Delta t}=\boldsymbol{d}(t+\Delta t)$, and $\boldsymbol{F}_{t}=$ $\boldsymbol{F}(t)-(\boldsymbol{K}-2 \hat{\boldsymbol{M}}) \boldsymbol{d}(t)-\hat{\boldsymbol{M}} \boldsymbol{d}(t-\Delta t)$. Then, the algorithm procedure to solve the hybrid equation of motion is stated as follows:

2. Set the initial displacement $\boldsymbol{d}(0)$, velocity $\dot{\boldsymbol{d}}(0)$, and external force $\boldsymbol{F}(0)$.

3. Calculate the initial acceleration $\ddot{\boldsymbol{d}}(0)$ in terms of Eq. (A.10).

4. Select the time step $\Delta t$, satisfying $\Delta t<\Delta t_{c}$, where $\Delta t_{c}$ denotes the critical value to ensure the stability of solution.

5. Calculate $\boldsymbol{d}(-\Delta t)$ in terms of Eq. (A.9).

6. Calculate $\hat{\boldsymbol{M}}=\boldsymbol{M} / \Delta t^{2}$.

7. For every time step $(t=0, \Delta t, 2 \Delta t, \cdots)$, calculate the effective loading $\boldsymbol{F}_{t}=\boldsymbol{F}(t)-(\boldsymbol{K}-2 \hat{\boldsymbol{M}}) \boldsymbol{d}(t)-$ $\hat{\boldsymbol{M}} \boldsymbol{d}(t-\Delta t)$.

8. Solve the linear equation Eq. (A.11), to get the displacement solution $\boldsymbol{d}_{t+\Delta t}$ at time $t+\Delta t$.

9. If needed, calculate acceleration $\ddot{\boldsymbol{d}}(t)$ and velocity $\dot{\boldsymbol{d}}(t)$ at time $t$ in terms of Eqs. (A.6) and (A.8).

10. If $t$ is less than the final time, then $t=t+\Delta t$ and return to the step 7. Otherwise, end the calculation.

\section{References}

1. Silling SA (2000) Reformulation of elasticity theory for discontinuities and long-range forces. Journal of the Mechanics and Physics of Solids 48(1):175-209

22

23

5

1
. ,

3

4

5

7

238

39


2. Silling SA, Lehoucq RB (2010) Peridynamic theory of solid mechanics. Advances in Applied Mechanics 44:73-168

3. Silling SA, Askari E (2005) A meshfree method based on the peridynamic model of solid mechanics. Computers \& Structures 83(17-18):1526-1535

4. Ha Y, Bobaru F (2010) Studies of dynamic crack propagation and crack branching with peridynamics. International Journal of Fracture 162(1-2):229-244

5. Silling S, Weckner O, Askari E, Bobaru F (2010) Crack nucleation in a peridynamic solid. International Journal of Fracture 162(12):219-227

6. Hu WK, Ha YD, Bobaru F (2012) Peridynamic model for dynamic fracture in unidirectional fiber-reinforced composites. Computer Methods in Applied Mechanics and Engineering 217:247-261

7. Hu WK, Ha YD, Bobaru F (2012) Peridynamic model for dynamic fracture in unidirectional fiber-reinforced composites. Computer Methods in Applied Mechanics and Engineering 217:247-261

8. F. Bobaru, J. T. Foster, P. H. Geubelle, and S. A. Silling, editors. Handbook of Peridynamic Modeling. Advances in Applied Mathematics. Chapman and Hall/CRC, 2016

9. Kilic B, Madenci E (2010) Coupling of peridynamic theory and the finite element method. Journal of Mechanics of Materials and Structures 5(5):707-733

10. Liu WY, Hong J-W (2012) A coupling approach of discretized peridynamics with finite element method. Computer Methods in Applied Mechanics and Engineering 245-246:163-75

11. Galvanetto U, Mudric T, Shojaei A, Zaccariotto M (2016) An effective way to couple FEM meshes and Peridynamics grids for the solution of static equilibrium problems. Mechanics Research Communications 76:41-47

12. Silling S, Littlewood D, Seleson P (2015) Variable horizon in a peridynamic medium. Journal of Mechanics of Materials and Structures 10(5):591-612

13. D'Elia M, Perego M, Bochev P, Littlewood D (2016) A coupling strategy for nonlocal and local diffusion models with mixed volume constraints and boundary conditions. Computers \& Mathematics with Applications 71(11):2218-2230

14. Han F, Lubineau G (2012) Coupling of nonlocal and local continuum models by the Arlequin approach. International Journal for Numerical Methods in Engineering 89(6):671-685

15. Lubineau G, Azdoud Y, Han F, Rey C, Askari A (2012) A morphing strategy to couple non-local to local continuum mechanics. Journal of the Mechanics and Physics of Solids 60(6):1088-1102

16. Seleson P, Beneddine S, Prudhomme S (2013) A force-based coupling scheme for peridynamics and classical elasticity. Computational Materials Science 66:34-49

17. Azdoud Y, Han F, Lubineau G (2013) A morphing framework to couple non-local and local anisotropic continua. International Journal of Solids and Structures 50(9):1332-1341

18. Azdoud Y, Han F, Lubineau G (2014) The morphing method as a flexible tool for adaptive local/non-local simulation of static fracture. Computational Mechanics 54(3):711-722

19. Han F, Lubineau G, Azdoud Y, Askari A (2016) A morphing approach to couple state-based peridynamics with classical continuum mechanics. Computer Methods in Applied Mechanics and Engineering 301:336-358
20. Han F, Lubineau G, Azdoud Y (2016) Adaptive coupling between damage mechanics and peridynamics: A route for objective simulation of material degradation up to complete failure. Journal of the Mechanics and Physics of Solids 94:453-472

21. Wang Y, Han F, Lubineau G (2019) A hybrid local/nonlocal continuum mechanics modeling and simulation of fracture in brittle materials. Computer Modeling in Engineering and Sciences 121(2):399-423

22. A. Askari, Y. Azdoud, F. Han, G. Lubineau, and S. Silling. 12 - Peridynamics for analysis of failure in advanced composite materials, pages 331-350. Woodhead Publishing, 2015

23. Y. Azdoud, F. Han, D. J. Littlewood, G. Lubineau, and P. Seleson. Chapter 14 Coupling Local and Nonlocal Models, book chapter 14, pages 405-436. Modern Mechanics and Mathematics. Taylor \& Francis/CRC Press, 2016

24. J. Fish, Nuggehally M. A., Shephard M. S., Picu C. R., Badia S., Parks M. L., and Gunzburger M. Concurrent atc coupling based on a blend of the continuum stress and the atomistic force. Computer Methods in Applied Mechanics and Engineering, 196(45):45484560, 2007

25. Bauman PT, Dhia HB, Elkhodja N, Oden JT, Prudhomme S (2008) On the application of the Arlequin method to the coupling of particle and continuum models. Computational Mechanics 42(4):511-530

26. Curtin WA, Miller RE (2003) Atomistic/continuum coupling in computational materials science. Modelling and Simulation in Materials Science and Engineering 11(3):R33-R68

27. Silling SA, Lehoucq RB (2008) Convergence of peridynamics to classical elasticity theory. Journal of Elasticity 93:13-37

28. Giannakeas IN, Papathanasiou TK, Bahai H (2019) Wave reflection and cut-off frequencies in coupled fe-peridynamic grids. International Journal for Numerical Methods in Engineering 120(1):29-55

29. Seleson P, Parks ML (2011) On the role of the influence function in the peridynamic theory. International Journal for Multiscale Computational Engineering 9(6):689-706

30. Ren HL, Zhuang XY, Rabczuk T (2017) Dual-horizon peridynamics: A stable solution to varying horizons. Computer Methods in Applied Mechanics and Engineering 318:762-782

31. Zaccariotto M, Mudric T, Tomasi D, Shojaei A, Galvanetto U (2018) Coupling of FEM meshes with Peridynamic grids. Computer Methods in Applied Mechanics and Engineering 330:471497

32. Xiang M, Hu H, Chen J (2013) Spalling and melting in nanocrystalline $\mathrm{Pb}$ under shock loading: Molecular dynamics studies. Journal of applied physics 113:144312

33. Xiang M, Chen J (2016) Numerical simulation of ductile fracture based on mean field homogenization method: Modeling and implementation. Engineering Fracture Mechanics 152:147-161

Publisher's Note Springer Nature remains neutral with regard to jurisdictional claims in published maps and institutional affiliations. 